\title{
Método Linear Bayesiano
}

\section{Péricles César de Araújo}

\author{
DISSERTAÇÃO APRESENTADA \\ $\mathrm{AO}$ \\ INSTITUTO DE MATEMÁTICA E ESTATÍSTICA \\ DA \\ UNIVERSIDADE DE SÃO PAULO \\ PARA OBTENÇÃO DO GRAU \\ $\mathrm{DE}$ \\ MESTRE EM ESTATÍSTICA \\ Área de Concentração: Estatística \\ Orientador: Prof. Dr. Sergio Wechsler
}

-São Paulo, 15 de julho de 1997- 
O tempo não pára. 


\section{Resumo}

Descrevemos o modelo de regressão linear sob um ponto de vista bayesiano, considerando os argumentos do Método Linear Bayesiano. O Método Linear Bayesiano é um processo recursivo que segue o esquema bayesiano, mas usa somente os primeiros e segundos momentos das distribuições envolvidas, sem requerer uma completa caracterização do modelo de probabilidade. Mostramos que o Filtro de Kalman é um caso particular do Método Linear Bayesiano.

\section{Abstract}

We describe the linear regression model from a Bayesian viewpoint by considering the Linear Bayesian Method. The Linear Bayesian Method is an iterative process which follows the bayesian scheme, but uses only the first two moments of the distributions involved, without demand for the complete probability model. We show that the Kalman Filter is a particular case of the Linear Bayesian Method. 


\section{Agradecimentos}

À Universidade de São Paulo, a oportunidade de poder melhorar a minha formação acadêmica.

Ao Prof.Dr. Sergio Wechsler, a orientação desta dissertação.

A todos os meus amigos e colegas, a convivência e pelo estímulo no desenvolvimento deste trabalho.

Aos Professores da UEFS, o incentivo e atenção.

Aos Professores do Departamento de Estatística da USP, a dedicação ao ensino.

Aos meus familiares, o amor e amizade constantes. 


\section{Índice}

$\begin{array}{ll}\text { Introdução } & 1\end{array}$

1 Regressão 5

2 Método Linear Bayesiano $\quad 19$

2.1 Introdução . . . . . . . . . . . . . . . . . . . . 19

2.2 Método Linear Bayesiano . . . . . . . . . . . . . . . 20

2.3 Método Linear Bayesiano Multivariado . . . . . . . . . . . . 35

2.4 Regressão . . . . . . . . . . . . . . . . . . . . . 36

3 Caracterização do Filtro de Kalman através do Método Linear Bayesiano

3.1 Introdução . . . . . . . . . . . . . . . . . . . . . . 38

3.2 Modelo de Filtro de Kalman . . . . . . . . . . . . . . . . . . . . . . . 39

3.3 Processo Iterativo de Estimação . . . . . . . . . . . . . . . . . . . . 39

3.4 Determinação da Distribuição Posteriori através da Normal . . . . . . 41

3.5 Caracterização do Filtro de Kalman através do Método Linear Bayesiano 45

3.6 Um Exemplo Numérico . . . . . . . . . . . . . . . . . . . . . . . . 48

$\begin{array}{lr}\text { Bibliografia } & \mathbf{5 0}\end{array}$ 


\section{Introdução}

O método bayesiano é representado pelo trio "conhecimento, experiência e opinião"como afirmou Pereira (1995) e segundo o Teorema de Bayes:

$$
p(\theta / x, H)=\frac{p(\theta / H) p(x / \theta, H)}{p(x / H)},
$$

onde $\theta$ é uma quantidade de interesse desconhecida (não observável), $H$ é a história e $x$ é a quantidade aleatória observada como definiram Gamerman e Migon (1993). Como

$$
p(x / H)=\int_{\Theta} p(x, \theta / H) d F(\theta / H)
$$

é uma constante, logo

$$
p(\theta / x, H) \propto p(x / \theta, H) p(\theta / H),
$$

onde $\propto$ denota proporcionalidade. Através do teorema de Bayes identificamos que o $p(\theta / x, H)$ (conhecimento) é obtido quando nós temos a $p(x / \theta, H)$ (experiência) e $p(\theta / H)$ (a sua opinião, construída através do Princípio Fundamental de Coerência Loschi,1992 ), como $H$ (a história) é comum a todos os termos, "pode ser removida mas não esquecida". De uma maneira mais usual o teorema de Bayes é

$$
p(\theta / x) \propto f(x / \theta) p(\theta)
$$

onde $f(x / \theta)$ é verossimilhança e $p(\theta)$ é a distribuiçao à priori do parâmetro. Portanto, o método Bayesiano requer uma completa caracterização do modelo de probabilidade. 


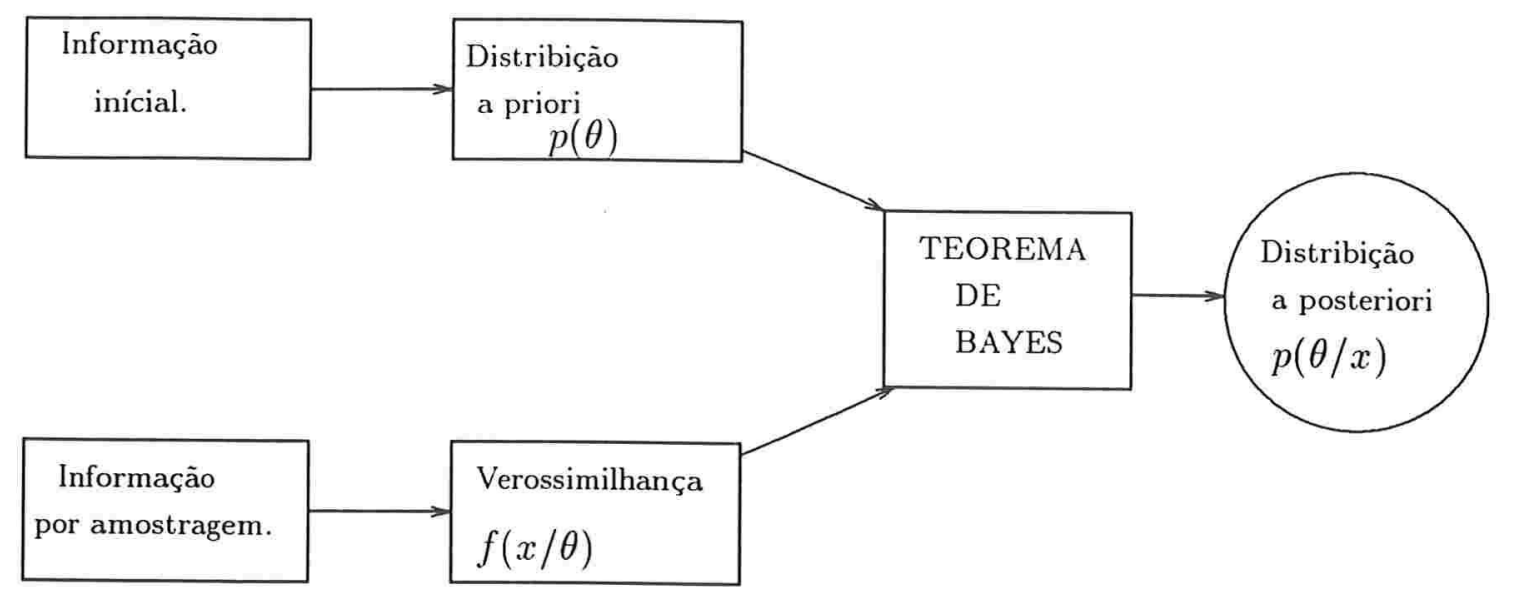

Como foi definido e está esquematizado acima, a densidade a posteriori $p(\theta / x)$ depende do experimento somente através da função de verossimilhança $f(x / \theta)$, desta forma, toda a inferência bayesiana é feita sobre $p(\theta / x)$, assim, podemos concluir que o Princípio da Verossimilhança (Dois resultados experimentais $x$ e y possuem a mesma informação sobre $\theta$ se $f(\theta / x)$ e $f(\theta / y)$ são proporcionais, Pereira e Viana(1982).) é uma conseqüência imediata do enfoque bayesiano, como ressaltou Inoue (1995).

Como observaram Aitchison e Dunsmore (1975), o objetivo final do argumento Bayesiano é a função densidade preditiva $p(y / x)$, isto é, o conceito da densidade de probabilidade de uma futura observação $y$ dado o resultado da informaçao do experimento $x$, que é representada pela a expressão:

$$
\begin{aligned}
p(y / x) & =\int_{\Theta} p(y / \theta) p(\theta / x) d \theta \\
& =\frac{\int_{\Theta} p(y / \theta) p(x / \theta) p(\theta) d \theta}{\int_{\Theta} p(x / \theta) p(\theta) d \theta} .
\end{aligned}
$$

No caso dos métodos não-bayesianos paramétricos onde é utilizada a hipótese do vetor de parâmetro ser fixo e desconhecido, esta hipótese não exige um conhecimento total do modelo de probabilidade, esta hipótese juntamente com o conceito frequentista de probabilidade pode ser vista como a causa fundamental para arbitrária parametrização, como afirmou Wechsler(1993). Desta maneira há um desconhecimento da distribuição à priori, assim como no não-paramétrico clássico a distribuição da população não é conhecida. 
Usualmente quando estamos interessados em aproximar uma curva de regessão através de uma função utilizamos o método dos mínimos quadrados. O método dos mínimos quadrados propõe que a melhor função que representa $E(Y / X=x)$ é a função $\zeta(x)$ que minimiza o valor esperado do quadrado do erro,

$$
\min E\left[(Y-E(Y / X))^{2}\right] \text {. }
$$

No Capítulo 1, analisamos o conceito de regressão e chamamos a atenção para os coeficientes da reta de regressão que são determinados através dos momentos de primeira e de segunda ordem.

No Capítulo 2, através dos argumentos de Hartigan (1969), construimos o Método Linear Bayesiano utilizando a definição de esperança e variância linear, suas propriedades e Teorema de Bayes para Esperança Linear. Verificamos que o Método Linear Bayesiano tem um grande eficiência computacional e não apresenta problemas com a parametrização, visto que a Esperança Linear não exige uma completa técnica de probabilidade necessitando somente do primeiro e segundo momentos da distribuição para processar uma previsão. A característica recursiva do Método Linear Bayesiano revela uma propriedade, que é comum ao método bayesiano, de atualização de nossas expectativas através de novas informações. A característica iterativa do Método Linear Bayesiano é implementada através do Teorema de Bayes para a Esperança Linear, que demonstramos no Teorema 2.2, e representamos por

$$
\begin{aligned}
\hat{V}^{-1}\left(Y / X, X_{n+1}\right) & =c^{2} \hat{V}^{-1}\left(X_{n+1} / Y, X\right)+\hat{V}^{-1}(Y / X), \\
\hat{V}^{-1}\left(Y / X, X_{n+1}\right) \hat{E}\left(Y / X, X_{n+1}\right) & =c \hat{V}^{-1}\left(X_{n+1} / Y, X\right)\left(X_{n+1}-d\right) \\
& +\hat{V}^{-1}(Y / X) \hat{E}(Y / X) .
\end{aligned}
$$

Os conceitos de esperança linear e variância, viabilizam a aplicação da versão linear do Teorema de Bayes, combinando

$$
\begin{gathered}
\hat{E}(Y / X) \text { e } \hat{V}^{-1}(Y / X)(\text { priori }) \\
\hat{E}\left(X_{n+1} / Y, X\right) \text { e } \hat{V}^{-1}\left(X_{n+1} / Y, X\right) c^{2}(\text { dados presentes }) \\
\hat{E}\left(Y / X, X_{n+1}\right) \text { e } \hat{V}^{-1}\left(Y / X, X_{n+1}\right)(\text { posteriori }) .
\end{gathered}
$$

No Capítulo 3, consideramos o artigo do Meinhold e Singpurwalla (1983), definimos o Filtro de Kalman do ponto de vista bayesiano e caracterizamos através do 
Método Linear Bayesiano.Demonstramos no Teorema 3.1 que podemos escrever o Filtro de Kalman exatamente como no Teorema de Bayes para a Esperança Linear

$$
\begin{aligned}
\hat{V}^{-1}\left(\theta_{t} / Y_{t}\right) & =\frac{F_{t}^{2}}{\hat{V}\left(Y_{t} / \theta_{t}, Y_{t-1}\right)}+\frac{1}{\hat{V}\left(Y_{t} / \theta_{t}\right)}, \\
\hat{V}^{-1}\left(\theta_{t} / Y_{t}\right) \hat{E}\left(\theta_{t} / Y_{t}\right) & =\frac{\hat{E}\left(\theta_{t} / Y_{t-1}\right)}{\hat{V}\left(\theta_{t} / Y_{t-1}\right)}+\frac{F_{t}}{\hat{V}\left(Y_{t} / \theta_{t}, Y_{t-1}\right)} Y_{t} .
\end{aligned}
$$

Mostramos também, que os conceitos de esperança linear e variância, viabilizam a aplicação da versão linear do Teorema de Bayes no Filtro de Kalman, combinando

$$
\begin{gathered}
\hat{E}\left(\theta_{t} / Y_{t-1}\right) \text { e } \hat{V}\left(\theta_{t} / Y_{t-1}\right)(\text { priori }), \\
\hat{E}^{-1}\left(Y_{t} / \theta, Y_{t-1}\right) e \hat{V}^{-1}\left(Y_{t} / \theta, Y_{t-1}\right) F_{t}^{2}(\text { dados presentes }), \\
\left.\hat{E}\left(\theta_{t} / Y_{t}\right) e \hat{V}^{-1}\left(\theta_{t} / Y_{t}\right) \text { (posteriori }\right) .
\end{gathered}
$$

Com estes resultados, onde $d=0$ e $c=F_{t}$, concluimos que o Filtro de Kalman é um caso particular do Método Linear Bayesiano. 


\section{Capítulo 1}

\section{Regressão}

Seja $(X, Y)$ um vetor aleatório de distribuição não-singular no plano (com todos os momentos de segunda ordem finitos). Para todo par $\left(a_{0}, a_{1}\right) \in \mathbb{R}^{2}$, definimos o seu "resíduo quadrático esperado" por:

$$
S\left(a_{0}, a_{1}\right)=E\left[\left(Y-a_{0}-a_{1} X\right)^{2}\right] .
$$

A " reta de regressão linear da distribuição conjunta de $(X, Y)$ " ou "regressão linear de $Y$ sobre $X$ " é definida como a reta que minimiza o resíduo quadrático esperado, isto é, a que atinge

$$
\inf _{a_{0}, a_{1}} E\left[\left(Y-a_{0}-a_{1} X\right)^{2}\right]
$$

e será denotada por $y=\alpha_{0}+\alpha_{1} x$.

No caso multivariado, ou seja, para $\left(Y, X_{1}, X_{2}, \ldots, X_{n}\right)$ de distribuição não-singular em $\mathbb{R}^{n+1}$ (com momentos de segunda ordem finitos), definimos a "regressão linear de $Y$ sobre $\left(X_{1}, X_{2}, \ldots, X_{n}\right)$ " como o hiperplano que atinge

$$
\inf _{a_{0}, a_{1}, \ldots, a_{n}} E\left[\left(Y-a_{0}-a_{1} X_{1}-\ldots-a_{n} X_{n}\right)^{2}\right]
$$

sendo denotado por

$$
y=\alpha_{0}+\sum_{i=1}^{n} \alpha_{i} x_{i}
$$


Teorema 1.1 Seja $(X, Y)$ um vetor aleatório no plano (de distribuição não-singular e com momentos de segunda ordem finitos ). Então, os coeficientes da reta de regressão linear

$$
y=\alpha_{0}+\alpha_{1} x
$$

são determinados pelos momentos de primeira e segunda ordem das distribuições envolvidas, da seguinte forma:

$$
\begin{aligned}
\alpha_{0} & =\frac{E(Y) E\left(X^{2}\right)-E(X) E(X Y)}{\operatorname{Var}(X)}=E(Y)-\rho \frac{\sigma_{y}}{\sigma_{x}} E(X) e \\
\alpha_{1} & =\frac{\operatorname{cov}(X, Y)}{\operatorname{Var}(X)}=\rho \frac{\sigma_{y}}{\sigma_{x}},
\end{aligned}
$$

onde

$$
\begin{aligned}
\sigma_{x} & =\sqrt{\operatorname{Var}(X)}=\sqrt{E\left[(X-E(X))^{2}\right]} \\
\sigma_{y} & =\sqrt{\operatorname{Var}(Y)}=\sqrt{E\left[(Y-E(Y))^{2}\right]} \\
\operatorname{cov}(X, Y) & =E[(X-E(X))(Y-E(Y))] \\
& e \\
\rho & =\frac{\operatorname{cov}(X, Y)}{\sigma_{x} \sigma_{y}} .
\end{aligned}
$$

Prova: Devemos minimizar

$$
S\left(a_{0}, a_{1}\right)=E\left[\left(Y-a_{0}-a_{1} X\right)^{2}\right] .
$$

As derivadas parciais são dadas por:

$$
\begin{aligned}
& \frac{\partial S\left(a_{0}, a_{1}\right)}{\partial a_{0}}=-2 E\left[Y-a_{0}-a_{1} X\right] e \\
& \frac{\partial S\left(a_{0}, a_{1}\right)}{\partial a_{1}}=-2 E\left[\left(Y-a_{0}-a_{1} X\right) X\right] .
\end{aligned}
$$

Deste modo, temos as equações normais: 


$$
\left\{\begin{array}{c}
E\left[Y-\alpha_{0}-\alpha_{1} X\right]=0 \\
E\left[\left(Y-\alpha_{0}-\alpha_{1} X\right) X\right]=0
\end{array}\right.
$$

ou

$$
\left\{\begin{array}{c}
E(Y)=\alpha_{0}+\alpha_{1} E(X) \\
E(X Y)=\alpha_{0} E(X)+\alpha_{1} E\left(X^{2}\right)
\end{array}\right.
$$

Finalmente, a regra de Cramer fornece o resultado.

O teorema acima caracteriza de forma única a reta de regressão linear de $Y$ sobre $X$, em função dos momentos de primeira e segunda ordem da distribuição de $(X, Y)$. Simetricamente, podemos considerar a reta de regressão linear de $X$ sobre $Y$, cujos coeficientes são

$$
\begin{aligned}
& \beta_{0}=E(X)-\rho \frac{\sigma_{x}}{\sigma_{y}} E(Y) e \\
& \beta_{1}=\rho \frac{\sigma_{x}}{\sigma_{y}} .
\end{aligned}
$$

Esta reta, $x=\beta_{0}+\beta_{1} y$, minimiza o resíduo $E\left[\left(X-b_{0}-b_{1} Y\right)^{2}\right]$. É natural considerar o menor ângulo formado pela duas retas como uma medida de associação linear entre as distribuições $X$ e $Y$. O teorema seguinte, de fato, formaliza esta noção.

Teorema 1.2 (Yule e Kendall[1945,p.226]) O menor ângulo formado pelas retas de regressão linear é dado por:

$$
\theta=\operatorname{arctg}\left[\frac{\left(1-\rho^{2}\right)}{|\rho|} \frac{\sigma_{x} \sigma_{y}}{\left(\sigma_{x}^{2}+\sigma_{y}^{2}\right)}\right]
$$




\section{Prova:}

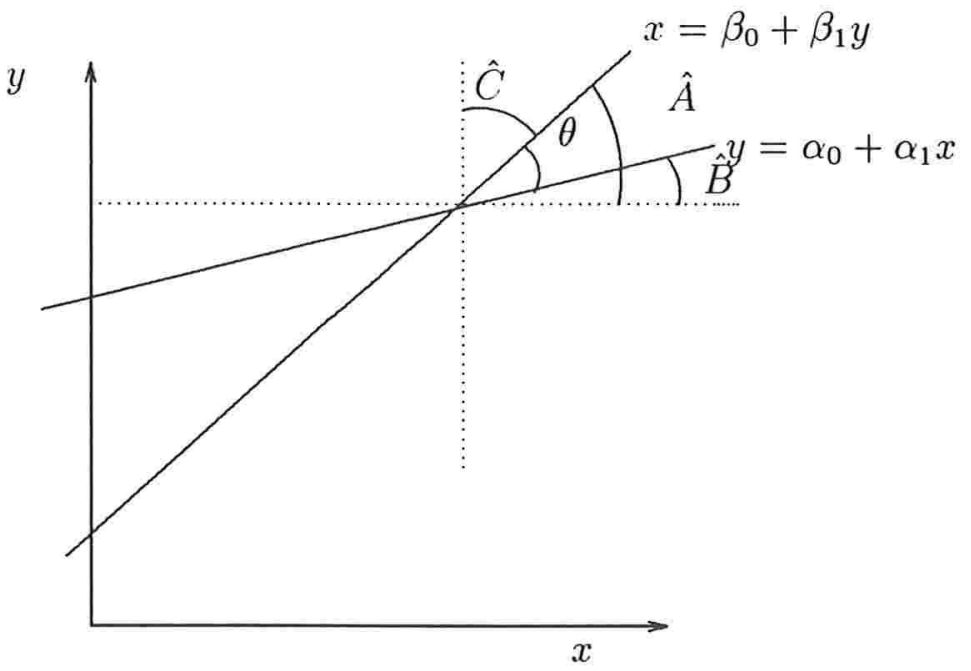

Na figura acima, temos, do Teorema 1.1,

$$
\begin{aligned}
\operatorname{tg} \hat{B} & =\rho \frac{\sigma_{y}}{\sigma_{x}} \\
e & \\
\operatorname{tg} \hat{C} & =\rho \frac{\sigma_{x}}{\sigma_{y}} .
\end{aligned}
$$

Por outro lado, como

$$
\operatorname{arctg}|\nu|+\operatorname{arctg} \frac{1}{|\nu|}=\frac{\pi}{2},
$$

temos

$$
|\hat{A}|=\frac{\pi}{2}-|\hat{C}|=\operatorname{arctg} \frac{1}{|\operatorname{tg} \hat{C}|}=\operatorname{arctg} \frac{\sigma_{y}}{|\rho| \sigma_{x}}
$$

de modo que

$$
\operatorname{tg}|\hat{A}|=\frac{\sigma_{y}}{|\rho| \sigma_{x}} .
$$


Portanto,

$$
\begin{aligned}
\operatorname{tg} \theta & =\operatorname{tg}|\hat{A}-\hat{B}|=\frac{|\operatorname{tg} \hat{A}|-|\operatorname{tg} \hat{B}|}{1+|\operatorname{tg} \hat{A}||\operatorname{tg} \hat{B}|} \\
& =\frac{\frac{\sigma_{y}}{|\rho| \sigma_{x}}-|\rho| \frac{\sigma_{y}}{\sigma_{x}}}{1+\frac{\sigma_{y}}{|\rho| \sigma_{x}}|\rho| \frac{\sigma_{y}}{\sigma_{x}}} \\
& =\frac{\left(1-\rho^{2}\right)}{|\rho|} \frac{\sigma_{x} \sigma_{y}}{\left(\sigma_{x}^{2}+\sigma_{y}^{2}\right)}
\end{aligned}
$$

Podemos observar que $\rho= \pm 1 \Rightarrow \theta=0$, e as retas são coincidentes, enquanto que $\rho=0 \Rightarrow \theta=\frac{\pi}{2}$, sendo as retas neste caso perpendiculares.

Teorema 1.3 O resíduo quadrático esperado da regressão linear de $Y$ sobre $X$ é

$$
S\left(\alpha_{0}, \alpha_{1}\right)=E\left\{\left(Y-\alpha_{0}-\alpha_{1} X\right)^{2}\right\}=\sigma_{y}^{2}\left(1-\rho^{2}\right)
$$

\section{Prova:}

Do Teorema 1.1,

$$
\begin{aligned}
S\left(\alpha_{0}, \alpha_{1}\right) & =E\left\{\left(Y-\alpha_{0}-\alpha_{1} X\right)^{2}\right\} \\
& =E\left\{\left[Y-E(Y)-\rho \frac{\sigma_{y}}{\sigma_{x}}(X-E(X))\right]^{2}\right\} \\
& =E\left\{[Y-E(Y)]^{2}-2 \rho \frac{\sigma_{y}}{\sigma_{x}}[X-E(X)][Y-E(Y)]+\right. \\
& \left.+\rho^{2} \frac{\sigma_{y}^{2}}{\sigma_{x}^{2}}[X-E(X)]^{2}\right\} \\
& =\sigma_{y}^{2}-2 \rho \frac{\sigma_{y}}{\sigma_{x}} \rho \sigma_{x} \sigma_{y}+\rho^{2} \sigma_{y}^{2} \\
& =\sigma_{y}^{2}-2 \rho^{2} \sigma_{y}^{2}+\rho^{2} \sigma_{y}^{2} \\
& =\sigma_{y}^{2}-\rho^{2} \sigma_{y}^{2} \\
& =\sigma_{y}^{2}\left(1-\rho^{2}\right) .
\end{aligned}
$$

Portanto, $\sigma_{y}^{2}\left(1-\rho^{2}\right)$ é o menor resíduo quadrático esperado que uma reta pode atingir para a distribuição conjunta de $(X, Y)$. Em seguida, apresentaremos as generalizações para o caso multivariado dos Teoremas 1.1 e 1.3 , respectivamente. 
Teorema 1.4 Seja $\left(Y, X_{1}, \ldots, X_{n}\right)$ um vetor aleatório de distribuição não-singular no $\mathbb{R}^{n+1}$ com momentos de segunda ordem finitos. Então, os coeficientes da regressão linear

$$
y=\alpha_{0}+\sum_{i=1}^{n} \alpha_{i} x_{i}
$$

são determinados através dos momentos de primeira e segunda ordem das distribuições marginais, da seguinte forma:

$$
\begin{aligned}
\alpha_{0} & \left.=E(Y)-\Sigma_{Y X} \Sigma_{X}^{-1} E \underset{\sim}{X}\right) \\
& e \\
\left(\alpha_{1}, \ldots, \alpha_{n}\right) & =\Sigma_{Y X} \Sigma_{X}^{-1}
\end{aligned}
$$

onde

$$
\begin{aligned}
\underset{\sim}{E} \underset{\sim}{X}) & =\left[\begin{array}{c}
E\left(X_{1}\right) \\
\vdots \\
E\left(X_{n}\right)
\end{array}\right]=\left[E\left(X_{i}\right)\right]_{n x 1}, \\
\Sigma_{Y X} & =\left[\operatorname{cov}\left(Y, X_{1}\right), \ldots, \operatorname{cov}\left(Y, X_{n}\right)\right]=\left[\operatorname{cov}\left(Y, X_{i}\right)\right]_{1 x n}
\end{aligned}
$$

\section{Prova:}

Vamos minimizar

$$
S\left(a_{0}, \ldots, a_{n}\right)=E\left[\left(Y-a_{0}-\sum_{i=1}^{n} a_{i} X_{i}\right)^{2}\right] .
$$

Utilizando os resultados do Cálculo Diferencial, determinamos os $\alpha_{i}^{\prime} s$ que verificam o sistema

$$
\frac{\partial S}{\partial \alpha_{i}}=0, \text { para } i=0, \ldots, n
$$


ou

$$
-2 E\left[\left(Y-\alpha_{0}-\sum_{j=1}^{n} \alpha_{j} X_{j}\right) \frac{\partial z\left(\alpha_{0}, \ldots, \alpha_{n}\right)}{\partial \alpha_{i}}\right]=0, i=0,1, \ldots, n
$$

onde

$$
z\left(\alpha_{0}, \ldots, \alpha_{n}\right)=\alpha_{0}+\sum_{j=1}^{n} \alpha_{j} X_{j}
$$

Explicitamente, temos o sistema

$$
\left\{\begin{array}{l}
E\left[\left(Y-\alpha_{0}-\sum_{j=1}^{n} \alpha_{j} X_{j}\right) \cdot 1\right]=0 \\
\cdots \ldots \ldots \ldots \ldots \ldots \ldots \ldots \ldots \ldots \ldots \ldots \ldots \ldots \ldots \ldots \ldots \ldots \ldots \ldots \ldots \ldots \ldots \\
E\left[\left(Y-\alpha_{0}-\sum_{j=1}^{n} \alpha_{j} X_{j}\right) X_{n}\right]=0
\end{array}\right.
$$

ou

$$
\left\{\begin{array}{l}
E(Y)=\alpha_{0}+\sum_{j=1}^{n} \alpha_{j} E\left(X_{j}\right) \\
E\left(Y X_{i}\right)=\alpha_{0} E\left(X_{i}\right)+\alpha_{i} E\left(X_{i}^{2}\right)+\sum_{j \neq 1}^{n} \alpha_{j} E\left(X_{i} X_{j}\right) \\
E\left(Y X_{n}\right)=\alpha_{0} E\left(X_{n}\right)+\sum_{j=1}^{n-1} \alpha_{j} E\left(X_{j} X_{n}\right)+\alpha_{n} E\left(X_{n}^{2}\right) .
\end{array}\right.
$$

Fazendo na primeira equação

$$
\alpha_{0}=E(Y)-\sum_{j=1}^{n} \alpha_{j} E\left(X_{j}\right)
$$

e substituindo nas n equações restantes do sistema acima, obtemos

$$
\left\{\begin{array}{l}
\operatorname{cov}\left(Y, X_{1}\right)=\alpha_{1} \operatorname{Var}\left(X_{1}\right)+\sum_{j=2}^{n} \alpha_{j} \operatorname{cov}\left(X_{1}, X_{j}\right) \\
\operatorname{cov}\left(Y_{j}, X_{i}\right)=\alpha_{i} \operatorname{Var}\left(X_{i}\right)+\sum_{j \neq i}^{n} \alpha_{j} \operatorname{cov}\left(X_{i}, X_{j}\right) \\
\operatorname{cov}\left(Y, X_{n}\right)=\sum_{j=1}^{n-1} \alpha_{j} \operatorname{cov}\left(X_{n}, X_{j}\right)+\alpha_{n} \operatorname{Var}\left(X_{n}\right),
\end{array}\right.
$$


Finalmente, a solução do sistema de equações normais é dada por:

$$
\left\{\begin{array}{l}
\alpha_{0}=E(Y)-\Sigma_{Y X} \Sigma_{X}^{-1} E(\underset{\sim}{X}) \\
\left(\alpha_{1}, \ldots, \alpha_{n}\right)=\Sigma_{Y X} \Sigma_{X}^{-1}
\end{array}\right.
$$

Teorema 1.5 O resíduo quadrático esperado do hiperplano de regressão linear de $Y$ sobre $\left(X_{1}, \ldots, X_{n}\right)$ é :

$$
S\left(\alpha_{0}, \alpha_{1} \ldots, \alpha_{n}\right)=\sigma_{y}^{2}-\Sigma_{Y X} \Sigma_{X}^{-1} \Sigma_{Y X}^{\prime}
$$

Prova: Seja $\underset{\sim}{X}=\left(X_{1}, \ldots, X_{n}\right)^{\prime}$;

$$
\begin{aligned}
S\left(\alpha_{0}, \alpha_{1}, \ldots, \alpha_{n}\right) & =E\left(Y-\alpha_{0}-\sum_{i=1}^{n} \alpha_{i} X_{i}\right)^{2} \\
& =E\left\{\left[Y-E(Y)-\Sigma_{Y X} \Sigma_{X}^{-1} \underset{\sim}{X}-\underset{\sim}{\left.\underset{\sim}{X}))]^{2}\right\}}\right.\right. \\
& =\sigma_{y}^{2}-2 \Sigma_{Y X} \Sigma_{X}^{-1} \Sigma_{Y X}^{\prime}+\Sigma_{Y X} \Sigma_{X}^{-1} \Sigma_{Y X}^{\prime} \\
& =\sigma_{y}^{2}-\Sigma_{Y X} \Sigma_{X}^{-1} \Sigma_{Y X}^{\prime} .
\end{aligned}
$$

Seja $(X, Y)$ um vetor aleatório com distribuição e suporte no plano e momentos finitos de segunda ordem, com função de distribuição $F_{X Y}$. Definimos a "curva de esperanças condicionais" da seguinte forma:

$$
\begin{aligned}
E(Y / X=x): \mathbb{R} & \rightarrow \mathbb{R} \\
x & \mapsto E(Y / X=x)=\int y d F(y / x) .
\end{aligned}
$$

É natural indagarmos qual a conexão entre retas de regressão linear e curvas de esperança condicionais. Veremos adiante que há coincidência dos conceitos essencialmente quando a distribuição conjunta é Normal. Mas há uma conexão na situação geral, dada pelo Teorema 1.6 abaixo 
Teorema 1.6 A reta de regressão $y=\alpha_{0}+\alpha_{1} x$ minimiza a soma de quadrados ponderados pela distribuição de $X$, isto é, faz atingir

$$
\inf _{a_{0}, a_{1}} \int\left(E(Y / X=x)-a_{0}-a_{1} x\right)^{2} d F_{X}(x) .
$$

\section{Prova(caso absolutamente contínuo):}

Vamos mostrar que a reta de regressão $y=\alpha_{0}+\alpha_{1} x$ minimiza

$$
\begin{aligned}
\psi\left(a_{0}, a_{1}\right) & =\int\left[E(Y / X=x)-a_{0}-a_{1} x\right]^{2} d F_{X}(x)= \\
& =E\left[\left(E(Y / X)-a_{0}-a_{1} X\right)^{2}\right] \\
& =E\left[(E(Y / X))^{2}\right]-2 a_{0} E(Y)-2 a_{1} E(X Y)+a_{0}^{2} \\
& +2 a_{0} a_{1} E(X)+a_{1}^{2} E\left(X^{2}\right) .
\end{aligned}
$$

As derivadas parciais são

$$
\begin{aligned}
& \frac{\partial \psi\left(a_{0}, a_{1}\right)}{\partial a_{0}}=-2 E(Y)+2 a_{0}+2 a_{1} E(X) e \\
& \frac{\partial \psi\left(a_{0}, a_{1}\right)}{\partial a_{1}}=-2 E(X Y)+2 a_{0} E(X)+2 a_{1} E\left(X^{2}\right)
\end{aligned}
$$

Desta forma, temos as equações normais:

$$
\left\{\begin{aligned}
E(Y) & =\alpha_{0}+\alpha_{1} E(X) e \\
E(X Y) & =\alpha_{0} E(X)+\alpha_{1} E\left(X^{2}\right),
\end{aligned}\right.
$$

iguais às obtidas na demonstração Teorema 1.1. Portanto, podemos concluir que a reta de regressão minimiza a soma de quadrados ponderados pela distribuição marginal de $X$.

Para passarmos à discussão do caso Normal, necessitamos antes do seguinte

Corolário 1.1 Seja $(X, Y)$ um vetor aleatório com distribuição não-singular em $\mathbb{R}^{2}$ e momentos de segunda ordem finitos. Se $E(Y / X=x)$ e $E(X / Y=y)$ são funções lineares (ou constantes) em $x$ e $y$, respectivamente, então $E(Y / X)=\alpha_{0}+\alpha_{1} X$ quase certamente e $E(X / Y)=\beta_{0}+\beta_{1} Y$ quase certamente. 
Prova: Por hipótese, temos

$$
\begin{array}{ll}
E(Y / X=x) & =a+b x \\
& e \\
E(X / Y=y) & =c+d y
\end{array}
$$

para valores determinados $a, b, c, d \in \mathbb{R}$. Ora, pelo Teorema 1.6, obtemos

$$
\begin{aligned}
& \inf _{a_{0}, a_{1}} \int\left[E(Y / X=x)-a_{0}-a_{1} x\right]^{2} d F_{X}(x)= \\
& =\int\left[E(Y / X=x)-\alpha_{0}-\alpha_{1} x\right]^{2} d F_{X}(x)
\end{aligned}
$$

ou

$$
\begin{aligned}
& \inf _{a_{0}, a_{1}} \int\left[a+b x-a_{0}-a_{1} x\right]^{2} d F_{X}(x)= \\
& =\int\left[E(Y / X=x)-\alpha_{0}-\alpha_{1} x\right]^{2} d F_{X}(x),
\end{aligned}
$$

resultando

$$
\int\left[E(Y / X=x)-\alpha_{0}-\alpha_{1} x\right]^{2} d F_{X}(x)=0
$$

implicando $E(Y / X)-\alpha_{0}+\alpha_{1} X=0$ quase certamente. A prova é análoga para $E(X / Y)$.

Teorema 1.7 Se o vetor aleatório $(X, Y)$ possui distribuição conjunta Normal nãosingular, então:

$$
\begin{aligned}
& E(Y / X=x)=\alpha_{0}+\alpha_{1} x \\
& E(X / Y=y)=\beta_{0}+\beta_{1} y
\end{aligned}
$$

\section{Prova:}

$$
\begin{aligned}
& \text { Como } Y / X=x \sim \text { Normal }\left[E(Y)+\rho \frac{\sigma_{y}}{\sigma_{x}}(x-E(X)), \sigma_{y}^{2}\left(1-\rho^{2}\right)\right] \text {, temos } \\
& \qquad \begin{aligned}
E(Y / X=x) & =E(Y)+\rho \frac{\sigma_{y}}{\sigma_{x}}(x-E(X)) \\
& =\left[E(Y)-\rho \frac{\sigma_{y}}{\sigma_{x}} E(X)\right]+\rho \frac{\sigma_{y}}{\sigma_{x}} x
\end{aligned}
\end{aligned}
$$


Mas estes coeficientes coincidem com os obtidos no Teorema 1.6, onde

$$
\begin{aligned}
\alpha_{0} & =E(Y)-\rho \frac{\sigma_{y}}{\sigma_{x}} E(X) \\
& e \\
\alpha_{1} & =\rho \frac{\sigma_{y}}{\sigma_{x}} .
\end{aligned}
$$

Portanto, quando o vetor aleatório $(X, Y)$ possui distribuição conjunta Normal,

$$
E(Y / X=x)=\alpha_{0}+\alpha_{1} x
$$

A demonstração é análoga para $E(X / Y=y)$.

O teorema acima estabeleceu a coincidência entre esperanças condicionais e retas de regressão linear no caso de normalidade da distribuição conjunta. Temos, por outro lado, a normalidade de distribuições conjuntas essencialmente quando as esperanças condicionais são lineares (ver Corolário do Teorema1.6) e as distribuições condicionais possuem " erros idênticos".

Definição 1.1 [Kendall, Stuart (1973), pg.366] Dizemos que a distribuição condicional de $Y$, dado $X$, possui " erros idênticos" quando para todo $x \in \mathbb{R}$, a distribuição de $Y$, dado $X=x$, depende de $x$ exclusivamente através de $E(Y / X=x)$.

Exemplo 1.1 Se $(X, Y)$ possui distribuição Normal bivariada, como para todo $x$, $Y / X=x \sim$ Normal $\left[E(Y)+\rho \frac{\sigma_{y}}{\sigma_{x}}(x-E(X)), \sigma_{y}^{2}\left(1-\rho^{2}\right)\right]$, vemos que a distribuição de $Y$, dado $X$, possui erros idênticos.

\section{Temos agora o}

Teorema 1.8 [Kendall, Stuart (1973), pg.364] Seja $(X, Y)$ um vetor aleatório com distribuição no plano. Se $E(Y / X=x)$ e $E(X / Y=y)$ são ambas lineares em $x$ e $y$, respectivamente, e se as distribuições condicionais de $Y$, dado $X$, e de $X$, dado $Y$, possuem ambas erros idênticos, então $(X, Y)$ possui distribuição Normal, a não ser que: $X$ seja independente de $Y$; ou $X$ e $Y$ sejam funcionalmente relacionadas.

A demonstração do Teorema 1.8 foge dos objetivos deste trabalho e será omitida. O leitor interessado pode consultar Kendall e Stuart(1973), páginas 367/368, ou Kagan e Karpov (1972). Apresentaremos em seguida um exemplo ilustrativo da importância da condição de erros idênticos. 
Exemplo 1.2 Consideremos o vetor aleatório $(X, Y)$, onde $X \sim N(0,1)$ e

$$
Y / X=x \sim \frac{1}{2} N(x, 1)+\frac{1}{2} N(-x, 1)
$$

(Isto é, $Y$, dado $X=x$, é uma mistura de Normais, com peso $1 / 2$.). É fácil ver que $Y \sim N(0,2)$ e que

$$
X / Y=y \sim \frac{1}{2} N\left(\frac{1}{2} y, \frac{1}{2}\right)+\frac{1}{2} N\left(-\frac{1}{2} y, \frac{1}{2}\right),
$$

de modo que

$$
\begin{aligned}
& E(Y / X=x)=0, \\
& V(Y / X=x)=1+x^{2}, \\
& E(X / Y=y)=0, \\
& V(X / Y=y)=1 / 2+\frac{y^{2}}{4} .
\end{aligned}
$$

(Note-se a violação da condição de erros idênticos, pelas variâncias das distribuições condicionais.)

A distribuição conjunta das variáveis $X$ e $Y$ possui densidade

$$
f(x, y)=\frac{1}{4 \pi} \exp \left[-\frac{1}{2}\left(y^{2}-2 x y+2 x^{2}\right)\right]+\frac{1}{4 \pi} \exp \left[-\frac{1}{2}\left(y^{2}+2 x y+2 x^{2}\right)\right],
$$

$\operatorname{com} \operatorname{Cov}(X, Y)=0$. A distribuição conjunta de $(X, Y)$ não é, evidentemente, Normal bivariada, uma vez que a condição de erros idênticos não está satisfeita (embora as esperanças condicionais sejam ambas iguais a 0 ). As curvas de contorno da densidade de $(X, Y)$ estão na Figura 1.1. 


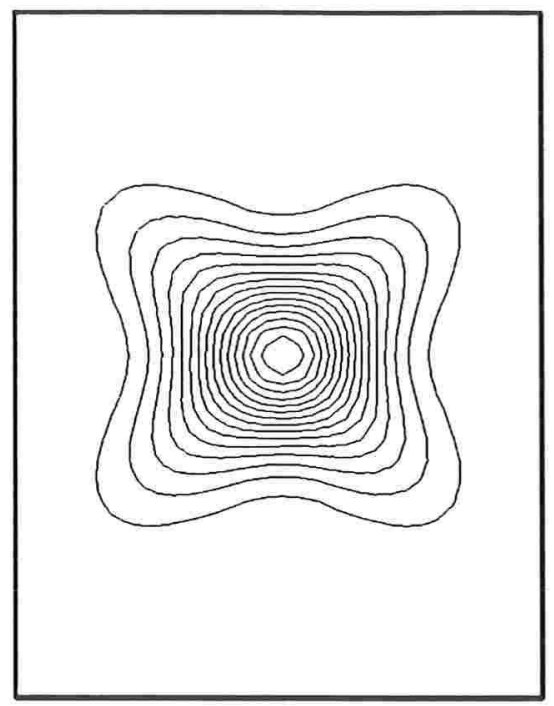

Figura 1.1: Contornos da densidade $f(x, y)$.

Notemos que $X$ e $Y$ tampouco são funcionalmente relacionadas ou independentes; a covariância entre $X$ e $Y$ é zero, mas com a ausência de normalidade conjunta, não se espera, de fato, que haja independência entre as marginais (ver James (1981), página 130). Temos, portanto, duas marginais normalmente distribuídas, não-correlacionadas, mas sem normalidade conjunta (e dependentes). A condição de erros idênticos é, evidentemente, violada (As variâncias condicionais dependem do valor condicionado.).

O teorema seguinte formaliza o fenômeno conhecido com "efeito de regressão". (Se $x$ é menor (ou maior) que sua média, $y$ tende também ser menor (ou maior) que sua média, porém mais próximo, em desvios-padrão.)

Teorema 1.9 Seja $(X, Y)$ um vetor aleatório no plano (com distribuição não-singular e momentos de segunda ordem finitos ). Com os coeficientes da reta de regressão linear $\alpha_{0}$ e $\alpha_{1}$ definidos de maneira usual, temos

$$
\left|\frac{x-E(X)}{\sigma_{x}}\right| \geq\left|\frac{\left(\alpha_{0}+\alpha_{1} x-E(Y)\right.}{\sigma_{y}}\right|,
$$

para todo $x \in \mathbb{R}$. 


\section{Prova:}

$$
\begin{aligned}
& \left|\frac{\alpha_{0}+\alpha_{1} x-E(Y)}{\sigma_{y}}\right|= \\
= & \left|\frac{E(Y)-\rho \frac{\sigma_{y}}{\sigma_{x}} E(X)+\rho \frac{\sigma_{y}}{\sigma_{x}} x-E(Y)}{\sigma_{y}}\right| \\
= & \left|\frac{-\rho \frac{\sigma_{y}}{\sigma_{x}} E(X)+\rho \frac{\sigma_{y}}{\sigma_{x}} x}{\sigma_{y}}\right| \\
= & \left|\frac{\rho[x-E(X)]}{\sigma_{x}}\right|=|\rho|\left|\frac{x-E(X)}{\sigma_{x}}\right| .
\end{aligned}
$$

Como $0 \leq|\rho| \leq 1$, o teorema está demonstrado. Podemos obter resultado equivalente quando temos a regressão

$$
x=\beta_{0}+\beta_{1} y
$$

de $X$ sobre $Y$. Neste caso, o efeito de regressão é caracterizado pela desigualdade

$$
\left|\frac{y-E(Y)}{\sigma_{y}}\right| \geq\left|\frac{\beta_{0}+\beta_{1} y-E(X)}{\sigma_{x}}\right| .
$$




\section{Capítulo 2}

\section{Método Linear Bayesiano}

\subsection{Introdução}

Em regressão linear, segundo Hartigan (1969), um conjunto de observações $Y_{1}, Y_{2}, \ldots, Y_{n}$ obedece o seguinte modelo:

$$
\begin{aligned}
E\left(Y_{i}\right) & =\sum_{i=1}^{m} a_{i, j} \mu_{j}, \\
\operatorname{Var}\left(Y_{i}\right) & =\sigma^{2}, \\
\operatorname{cov}\left(Y_{i}, Y_{j}\right) & =0 .
\end{aligned}
$$

Hartigan (1969) observa, também, que estas suposições envolvem somente o primeiro e o segundo momentos das observações dado os parâmetros. Analisaremos o modelo de regressão linear do ponto de vista bayesiano e considerando os argumentos propostos por Hartigan (1969), que exige o esquema bayesiano, mas usa somente o primeiro e o segundo momentos da distribuição, supondo, também, que as "prioris" dos parâmetros do primeiro e segundo momentos são conhecidas. Quando a hipótese de normalidade é utilizada em regressão linear, do ponto de vista clássico que é justificado pelo fato de a condicional de duas normais ser também normal, que a esperança e variância condicionais são funções lineares. Aqui usaremos o conceito de esperança e variância lineares que são equivalentes ao conceito clássico quando há suposição de normalidade. O conceito de esperança e variância lineares de Hartigan (1969), viabiliza a aplicação do teorema de Bayes, combinando a priori, conhecimento presente dos estimadores dos parâmetros e variância dos estimadores. 


\subsection{Método Linear Bayesiano}

Apresentamos agora as definições de esperança e variância linear propostas por Hartigan (1969). Sejam $\left(Y, X_{1}, X_{2}, \ldots, X_{n}\right)$ um vetor aleatório com momentos de segunda ordem finitos. Define-se a esperança linear de $Y$, dados $X_{1}, X_{2}, \ldots, X_{n}$ por

$$
\hat{E}\left(Y / X_{1}, \ldots, X_{n}\right)=\sum_{i=1}^{n} \alpha_{i} X_{i}+\alpha_{0},
$$

onde os $\alpha_{i}$ 's são os coeficientes do hiperplano da regressão linear de $Y$ sobre $X_{1}, \ldots, X_{n}$. A variância linear de $Y$, dados $X_{1}, X_{2}, \ldots, X_{n}$ é definida por

$$
\hat{V}\left(Y / X_{1}, \ldots, X_{n}\right)=S\left(\alpha_{0}, \ldots, \alpha_{n}\right)
$$

Sob a hipótese de normalidade conjunta, estas definições coincidem com as definições usuais de esperança e variância condicionais, como foi visto no final do Capítulo 1. Como uma distribuição Normal conjunta pode ser construída com apenas primeiros e segundos momentos, muitas das propriedades da esperança condicional ordinária e variância condicional são mantidas para a esperança e variância lineares. A seguir, analisaremos estas propriedades da esperança e variância lineares propostas por Hartigan (1969).

\section{Teorema 2.1}

1. $\hat{E}\left(X_{i} / X_{1}, \ldots, X n\right)=X_{i}$ para $i=1,2, \ldots, n$,

2. $\hat{E}\left(c / X_{1}, \ldots, X_{n}\right)=c$, para $c \in \mathbb{R}$.

3. Seja $c \neq 0$. Então, $\hat{E}\left(c Y / X_{1}, \ldots, X_{n}\right)=c \hat{E}\left(Y / X_{1}, . ., X_{n}\right)$.,

4. $\hat{E}\left(Y_{1}+Y_{2} / X_{1}, \ldots, X_{n}\right)=\hat{E}\left(Y_{1} / X_{1}, \ldots, X_{n}\right)+\hat{E}\left(Y_{1} / X_{1}, \ldots, X_{n}\right)$,

5. $\hat{E}\left(\hat{E}\left(Y / X_{1}, \ldots, X_{n+1}\right) / X_{1}, \ldots, X_{n}\right)=\hat{E}\left(Y / X_{1}, \ldots, X_{n}\right)$,

6. $\hat{V}(Y)=\hat{E}\left(\hat{V}\left(Y / X_{1}, \ldots, X_{n}\right)\right)+\hat{V}\left(\hat{E}\left(Y / X_{1}, \ldots, X_{n}\right)\right)$. 
Prova da propriedade 1:

$$
\begin{aligned}
\hat{E}\left(X_{i} / X_{1}, \ldots, X_{n}\right)= & \alpha_{0}+\alpha_{i} X_{i}+\sum_{j \neq i}^{n} \alpha_{j} X_{j}, \text { onde } \\
& E\left[\left(X_{i}-\alpha_{0}-\alpha_{i} X_{i}-\sum_{j \neq i}^{n} \alpha_{j} X_{j}\right)^{2}\right]
\end{aligned}
$$

é mínima. Ora, se $\alpha_{i}=1$ e $\alpha_{j}=0$ para $j \neq i$ e $\alpha_{0}=0$, temos

$$
E\left[\left(X_{i}-\alpha_{0}-\alpha_{i} X_{i}-\sum_{j \neq i}^{n} \alpha_{j} X_{j}\right)^{2}\right]=0
$$

que é mínimo. Logo, $\hat{E}\left(X_{i} / X_{1}, \ldots, X_{n}\right)=X_{i}$.

\section{Prova da propriedade 2:}

Fazendo $\alpha_{0}=c$ e $\alpha_{j}=0$ para $j=1,2, \ldots, n$, temos

$$
E\left[\left(c-\alpha_{0}-\sum_{j}^{n} \alpha_{j} X_{j}\right)^{2}\right]=0 .
$$

\section{Prova da propriedade 3:}

Basta perceber que, se

$$
\begin{aligned}
\hat{E}\left(Y / X_{1}, \ldots, X_{n}\right) & =\alpha_{0}+\sum_{i=1}^{n} \alpha_{i} X_{i} \\
E\left[\left(c Y-c \alpha_{0}-c \sum_{i=1}^{n} \alpha_{i} X_{i}\right)^{2}\right] & =c^{2} E\left[\left(Y-\alpha_{0}-\sum_{i=1} \alpha_{i} X_{i}\right)^{2}\right]
\end{aligned}
$$

que é mínima.

Prova da propriedade 4: Consideremos um vetor aleatório

$$
\left(T_{1}, T_{2}, V_{1}, \ldots, V_{n}\right)
$$

com distribuição Normal não-singular com os mesmos momentos de primeira e segunda ordem que o vetor aleatório $\left(Y_{1}, Y_{2}, X_{1}, \ldots, X_{n}\right)$. Então, como $\hat{E}\left(Y_{1}+Y_{2} / X_{1}, \ldots, X_{n}\right)$ depende apenas dos momentos de primeira e segunda ordem da distribuição conjunta, 
temos $\hat{E}\left(Y_{1}+Y_{2} / X_{1}, \ldots, X_{n}\right)=\hat{E}\left(T_{1}+T_{2} / V_{1}, \ldots, V_{n}\right)$. Mas, pela normalidade de $\left(T_{1}, T_{2}, V_{1}, \ldots, V_{n}\right)$ e suas marginais, temos:

$$
\begin{aligned}
\hat{E}\left(T_{1}+T_{2} / V_{1}, \ldots, V_{n}\right) & =E\left(T_{1}+T_{2} / V_{1}, \ldots, V_{n}\right) \\
& =E\left(T_{1} / V_{1}, \ldots, V_{n}\right)+E\left(T_{2} / V_{1}, \ldots, V_{n}\right) \\
& =\hat{E}\left(T_{1} / V_{1}, \ldots, V_{n}\right)+\hat{E}\left(T_{2} / V_{1}, \ldots, V_{n}\right) \\
& =\hat{E}\left(Y_{1} / X_{1}, \ldots, X_{n}\right)+\hat{E}\left(Y_{2} / X_{1}, \ldots, X_{n}\right) .
\end{aligned}
$$

Como consequência, temos

$$
\hat{E}\left(Y_{1}+Y_{2} / X_{1}, \ldots, X_{n}\right)=\hat{E}\left(Y_{1} / X_{1}, \ldots, X_{n}\right)+\hat{E}\left(Y_{2} / X_{1}, \ldots, X_{n}\right)
$$

\section{Prova da propriedade 5:}

Queremos demonstrar que

$$
\begin{aligned}
\hat{E}\left(\hat{E}\left(Y / X_{1}, \ldots, X_{n+1}\right) / X_{1}, \ldots, X_{n}\right) & =\hat{E}\left(Y / X_{1}, \ldots, X_{n}\right) \\
& \text { ou } \\
\hat{E}\left(\left[\alpha_{0}+\sum_{i=1}^{n} \alpha_{i} X_{i}+\alpha_{n+1} X_{n+1}\right] / X_{1}, \ldots, X_{n}\right) & =\hat{E}\left(Y / X_{1}, \ldots, X_{n}\right)
\end{aligned}
$$

onde $\left(\alpha_{0}, \alpha_{1}, \ldots, \alpha_{n+1}\right)$ são coeficientes da regressão de $Y$ dado $X_{1}, \ldots, X_{n+1}$. Utilizando os resultados das propriedades anteriores temos:

$$
\begin{aligned}
\alpha_{0}+\sum_{i=1}^{n} \alpha_{i} \hat{E}\left(X_{i} / X_{1}, \ldots, X_{n}\right)+\alpha_{n+1} \hat{E}\left(X_{n+1} / X_{1}, \ldots, X_{n}\right) & =\hat{E}\left(Y / X_{1}, \ldots, X_{n}\right) \\
\alpha_{0}+\sum_{i=1}^{n} \alpha_{i} X_{i}+\alpha_{n+1} \hat{E}\left(X_{n+1} / X_{1}, \ldots, X_{n}\right) & =\hat{E}\left(Y / X_{1}, \ldots, X_{n}\right) .
\end{aligned}
$$

Então,

$$
\begin{aligned}
\alpha_{0}+\sum_{i=1}^{n} \alpha_{i} X_{i} & =\hat{E}\left(Y-\alpha_{n+1} X_{n+1} / X_{1}, \ldots, X_{n}\right) \\
& =\beta_{0}+\sum_{i=1}^{n} \beta_{i} X_{i},
\end{aligned}
$$


onde $\left(\beta_{0}, \ldots, \beta_{n}\right)$ minimiza

$E\left\{\left[\left(Y-\alpha_{n+1} X_{n+1}\right)-b_{0}-\sum_{j=1}^{n} b_{j} X_{j}\right]^{2}\right\}=E\left\{\left[Y-\left(\alpha_{n+1} X_{n+1}+b_{0}-\sum_{j=1}^{n} b_{j} X_{j}\right)\right]^{2}\right\}$.

Ora, na expressão acima, fica evidente que $\beta_{0}=\alpha_{0}$ e $\beta_{j}=\alpha_{j}, j=1, \ldots, n$; pois $\left(\alpha_{0}, \alpha_{1}, \ldots, \alpha_{n+1}\right)$ minimiza $E\left\{\left(Y-a_{0}-\sum_{i=1}^{n} a_{i} X_{i}-a_{n+1} X_{n+1}\right)^{2}\right\}$. Daí o resultado.

\section{Prova da propriedade 6:}

Lema 2.1 Seja $Z$ um vetor aleatório $\operatorname{com} E(Z)<\infty$, então,

$$
\begin{aligned}
\hat{E}(Z) & =E(Z) \\
& e \\
\hat{V}(Z) & =\operatorname{Var}(Z)
\end{aligned}
$$

\section{Prova:}

Como

$$
\begin{aligned}
\mathcal{D} & =E(Z-a)^{2} \\
\frac{\partial \mathcal{D}}{\partial a} & =0 \Rightarrow E(Z)=\alpha_{0}=\hat{E}(Z)
\end{aligned}
$$

é fácil ver que $\hat{V}(Z)=V(Z)$.

Consideramos um vetor aleatório $\left(T, V_{1}, \ldots, V_{n}\right)$ com distribuição Normal nãosingular e momentos iguais ao do vetor aleatório $\left(Y, X_{1}, \ldots, X_{n}\right)$. Então, temos:

$$
\begin{aligned}
\hat{E}\left(\hat{V}\left(Y / X_{1}, \ldots, X_{n}\right)\right) & =E\left(\hat{V}\left(T / V_{1}, \ldots, V_{n}\right)\right) \\
& =E\left(V\left(T / V_{1}, \ldots, V_{n}\right)\right) .
\end{aligned}
$$

Analogamente,

$$
\hat{V}\left(\hat{E}\left(Y / X_{1}, \ldots, X_{n}\right)\right)=V\left(E\left(T / V_{1}, \ldots, V_{n}\right)\right) .
$$


Logo,

$$
\begin{aligned}
\hat{E}\left(\hat{V}\left(Y / X_{1}, \ldots, X_{n}\right)\right)+ & \hat{V}\left(\hat{E}\left(Y / X_{1}, \ldots, X_{n}\right)\right)= \\
& \operatorname{Var}(T)=\hat{V}(T)=\hat{V}(Y)=\operatorname{Var}(Y) .
\end{aligned}
$$

Teorema 2.2 (Teorema de Bayes para a Esperança Linear).

Seja $\hat{E}\left(X_{n+1} / Y, X\right)=c Y+d$, onde $X=\left(X_{1}, \ldots, X_{n}\right)$ e $d$ é da forma

$$
\alpha_{0}+\sum_{i=1}^{n} \alpha_{i} X_{i}
$$

Então,

$$
\begin{aligned}
\hat{V}^{-1}\left(Y / X, X_{n+1}\right) & =c^{2} \hat{V}^{-1}\left(X_{n+1} / Y, X\right)+\hat{V}^{-1}(Y / X), \\
\hat{V}^{-1}\left(Y / X, X_{n+1}\right) \hat{E}\left(Y / X, X_{n+1}\right) & =c \hat{V}^{-1}\left(X_{n+1} / Y, X\right)\left(X_{n+1}-d\right) \\
& +\hat{V}^{-1}(Y / X) \hat{E}(Y / X) .
\end{aligned}
$$

\section{Prova:}

Se $Y, X$ e $X_{n+1}$ fossem conjuntamente normais, seja $f(y / x)$ denotando a densidade condicional de $y$ dado $x$ e utilizando dos seguintes fatos $E(Y / X)=\hat{E}(Y / X)$ e $\operatorname{Var}(Y / X)=\hat{V}(Y / X)$. Então, temos que

$$
\begin{aligned}
f\left(y / x, x_{n+1}\right) & =\frac{f\left(y, x, x_{n+1}\right)}{f\left(x, x_{n+1}\right)}=\frac{f\left(x_{n+1} / y, x\right) f(y, x)}{f(x) f\left(x_{n+1} / x\right)} \\
& =\frac{f\left(x_{n+1} / y, x\right) f(y / x) f(x)}{f(x) f\left(x_{n+1} / x\right)}=\frac{f\left(x_{n+1} / y, x\right) f(y / x)}{f\left(x_{n+1} / x\right)}
\end{aligned}
$$


onde

$$
\begin{aligned}
f\left(y / x, x_{n+1}\right) & \propto \exp \left[-\frac{1}{2} \frac{\left(y-\hat{E}\left(Y / X, X_{n+1}\right)\right)^{2}}{\hat{V}\left(Y / X, X_{n+1}\right)}\right] \\
f\left(x_{n+1} / y, x\right) & \propto \exp \left[-\frac{1}{2} \frac{\left(x_{n+1}-\hat{E}\left(X_{n+1} / Y, X\right)\right)^{2}}{\hat{V}\left(X_{n+1} / Y, X\right)}\right], \\
f(y / x) & \propto \exp \left[-\frac{1}{2} \frac{(y-\hat{E}(Y / X))^{2}}{\hat{V}(Y / X)}\right], \\
f\left(x_{n+1} / x\right) & \propto \exp \left[-\frac{1}{2} \frac{\left(x_{n+1}-\hat{E}\left(X_{n+1} / X\right)\right)^{2}}{\hat{V}\left(X_{n+1} / X\right)}\right],
\end{aligned}
$$

do teorema de Bayes (Hartigan-1969),

$$
\log f\left(y / x, x_{n+1}\right)+\log f\left(x_{n+1} / x\right)=\log f\left(x_{n+1} / y, x\right)+\log f(y / x),
$$

temos:

$$
\begin{aligned}
& \frac{\left(y-\hat{E}\left(Y / X, X_{n+1}\right)^{2}\right.}{\hat{V}\left(Y / X, X_{n+1}\right)}+\frac{\left(x_{n+1}-\hat{E}\left(X_{n+1} / X\right)\right)^{2}}{\hat{V}\left(X_{n+1} / X\right)}= \\
& \frac{\left(x_{n+1}-\hat{E}\left(X_{n+1} / Y, X\right)\right)^{2}}{\hat{V}\left(X_{n+1} / Y, X\right)}+\frac{(y-\hat{E}(Y / X))^{2}}{\hat{V}(Y / X)} .
\end{aligned}
$$

Como $\hat{E}\left(X_{n+1} / Y, X\right)=c y+d$,

$$
\begin{aligned}
& \frac{\left(y-\hat{E}\left(Y / X, X_{n+1}\right)\right)^{2}}{\hat{V}\left(Y / X, X_{n+1}\right)}+\frac{\left(x_{n+1}-\hat{E}\left(X_{n+1} / X\right)\right)^{2}}{\hat{V}\left(X_{n+1} / X\right)}= \\
& \frac{\left(x_{n+1}-(c y+d)\right)^{2}}{\hat{V}\left(X_{n+1} / Y, X\right)}+\frac{(y-\hat{E}(Y / X))^{2}}{\hat{V}(Y / X)}, \\
& \frac{\left(y-\hat{E}\left(Y / X, X_{n+1}\right)\right)^{2}}{\hat{V}\left(Y / X, X_{n+1}\right)}+\frac{\left(x_{n+1}-\hat{E}\left(X_{n+1} / X\right)\right)^{2}}{\hat{V}\left(X_{n+1} / X\right)}= \\
& \frac{c^{2}\left(1 / c\left(x_{n+1}-d\right)-y\right)^{2}}{\hat{V}\left(X_{n+1} / Y, X\right)}+\frac{(y-\hat{E}(Y / X))^{2}}{\hat{V}(Y / X)},
\end{aligned}
$$


finalmente temos as seguintes equações:

$$
\begin{aligned}
& \frac{\left(y-\hat{E}\left(Y / X, X_{n+1}\right)\right)^{2}}{\hat{V}\left(Y / X, X_{n+1}\right)}=\frac{1}{\hat{V}\left(Y / X, X_{n+1}\right)} y^{2}-2 y \frac{\hat{E}\left(Y / X, X_{n+1}\right)}{\hat{V}\left(Y / X, X_{n+1}\right)}+\kappa_{1}, \\
& \frac{c^{2}\left(1 / c\left(x_{n+1}-d\right)-y\right)^{2}}{\hat{V}\left(X_{n+1} / Y, X\right)}=\frac{c^{2}}{\hat{V}\left(X_{n+1} / Y, X\right)} y^{2}-2 y \frac{c\left(x_{n+1}-d\right)}{\hat{V}\left(X_{n+1} / Y, X\right)}+\kappa_{2}, \\
& \frac{(y-\hat{E}(Y / X))^{2}}{\hat{V}(Y / X)}=\frac{1}{\hat{V}(Y / x)} y^{2}-2 y \frac{\hat{E}(Y / X)}{\hat{V}(Y / X)}+\kappa_{3},
\end{aligned}
$$

onde $\kappa_{1}, \kappa_{2}$ e $\kappa_{3}$ são constantes independentes de y. Como Hartigan(1969) afirmou, o coeficiente de $y^{2}$ na equação 2.2 é a variância linear, isto é:

$$
\frac{1}{\hat{V}\left(Y / X, X_{n+1}\right)}=\frac{c^{2}}{\hat{V}\left(X_{n+1} / Y, X\right)}+\frac{1}{\hat{V}(Y / X)}
$$

seguindo com o autor citado, os coeficientes de $y$ na equação é a esperança linear,

$$
\frac{\hat{E}\left(Y / X, X_{n+1}\right)}{\hat{V}\left(Y / X, X_{n+1}\right)}=\frac{c\left(X_{n+1}-d\right)}{\hat{V}\left(X_{n+1} / Y, X\right)}+\frac{\hat{E}(Y / X)}{\hat{V}(Y / X)}
$$

Os conceitos de esperança linear e variância, viabilizam a aplicação da versão linear do Teorema de Bayes, combinando

$$
\begin{gathered}
\hat{E}(Y / X) \text { e } \hat{V}^{-1}(Y / X)(\text { priori }), \\
\hat{E}\left(X_{n+1} / Y, X\right) \text { e } \hat{V}^{-1}\left(X_{n+1} / Y, X\right) c^{2}(\text { dados presentes }), \\
\hat{E}\left(Y / X, X_{n+1}\right) \text { e } \hat{V}^{-1}\left(Y / X, X_{n+1}\right)(\text { posteriori }) .
\end{gathered}
$$

Como foi verificado acima, com Teorema de Bayes para Esperança Linear, podemos observar que no contexto da Esperança Linear, proposto por Hartigan(1969), são preservados os fundamentos do método Bayesiano que é opinião que está representada pela

$$
\hat{E}(Y / X) \text { e } \hat{V}(Y / X)
$$


a experiência que está representada por

$$
\hat{E}\left(X_{n+1} / Y, X\right) \text { e } \hat{V}^{-1}\left(X_{n+1} / Y, X\right) c^{2}
$$

e finalmente temos o conhecimento representado por

$$
\hat{E}\left(Y / X, X_{n+1}\right) \text { e } \hat{V}^{-1}\left(Y / X, X_{n+1}\right) .
$$

Considerando os resultados acima, a Esperança Linear pode ser escrita da seguinte forma

$$
\hat{E}\left(Y / X, X_{n+1}\right)=\alpha_{0_{(n+1)}}+\alpha_{1_{(n+1)}} X_{n+1}
$$

onde

$$
\begin{aligned}
\alpha_{0_{(n+1)}} & =\frac{-c \hat{V}^{-1}\left(X_{n+1} / Y, X\right) d+\hat{V}^{-1}(Y / X) \hat{E}(Y / X)}{\hat{V}^{-1}\left(Y / X, X_{n+1}\right)} \\
& e \\
\alpha_{1_{(n+1)}} & =\frac{c \hat{V}^{-1}\left(X_{n+1} / Y, X\right)}{\hat{V}^{-1}\left(Y / X, X_{n+1}\right)} .
\end{aligned}
$$

Aqui o método da Esperança Linear não exige uma completa caracterização. Desta forma, não temos a distribuiçao a priori do parâmetro $p(\theta)$, assim como a distribuiçao condicional das observações dado o parâmetro $p(y / \theta)$. Outra característica que temos no método da Esperança Linear é que determinamos a esperança preditiva $\hat{E}\left(Y / X, X_{n+1}\right)$, que é o objetivo final do argumento Bayesiano, sem utilizarmos de uma completa técnica de probabilidade da função preditiva

$$
p\left(y / x, x_{n+1}\right)=\int_{\Theta} p(y / \theta) p\left(\theta / x, x_{n+1}\right) d \theta
$$

isto é, determinamos a Esperança Linear $\hat{E}\left(Y / X, X_{n+1}\right)$ sem precisar determinar $p(y / \theta)$ e $p\left(\theta / x, x_{n+1}\right)$, funções que envolvem o parâmetro $\theta$. Portanto, o método da Esperança Linear sugere que a previsão estatística, $\hat{E}\left(Y / X, X_{n+1}\right)$, é um processo iterativo onde a variável aleatória $Y$ é deduzida a partir das observações correntes $X$ e atualizada através de outras informações $X_{n+1}$.

A idéia de utilizar somente o primeiro e o segundo momentos das distribuições elimina as arbitrariedades causadas pela parametrização, que é o componente básico do processo de redução de deFinetti. Esta atitude de restringir a influência dos parâmetros é chamada de preditivismo (Wechsler, 1993).

Exemplo 2.1 Sejam $X_{1}, X_{2}, \mu$ variáveis aleatórias condicionalmente independentes e identicamente distribuídas, com: 


$$
\begin{aligned}
& E(\mu)=\mu_{0}, \\
& V(\mu)=E\left(\mu-\mu_{0}\right)^{2}=\sigma_{0}^{2}, \\
& E\left(X_{1}\right)=E\left(X_{1} / \mu\right)=\mu_{0}, \\
& V\left(X_{1} / \mu\right)=E(X-\mu)^{2}=\sigma^{2} .
\end{aligned}
$$

Das hipóteses acima, temos os seguintes resultados:
1. $\operatorname{Cov}\left(X_{1}, \mu\right)=\sigma_{0}^{2}$,
2. $\operatorname{Cov}\left(X_{1}, X_{2}\right)=\sigma_{0}^{2}$,
3. $V\left(X_{1}\right)=\sigma^{2}+\sigma_{0}^{2}$.

Prova do resultado 1:

$$
\begin{aligned}
\operatorname{Cov}\left(X_{1}, \mu\right) & =E\left(X_{1}, \mu\right)-E\left(X_{1}\right) E(\mu) \\
& =E\left(E\left(X_{1} \mu / \mu\right)\right)-\mu_{0}^{2} \\
& \left.=E\left(E\left(X_{1} / \mu\right) \mu\right)\right)-\mu_{0}^{2} \\
& =E\left(\mu^{2}\right)-\mu_{0}^{2} \\
& =\sigma_{0}^{2}
\end{aligned}
$$

Prova do resultado 2:

$$
\begin{aligned}
\operatorname{Cov}\left(X_{1}, X_{2}\right) & =E\left(X_{1}, X_{2}\right)-E\left(X_{1}\right) E\left(X_{2}\right) \\
& =E\left(E\left(X_{1} X_{2} / \mu\right)\right)-\mu_{0}^{2} \\
& =E\left(E\left(X_{1} / \mu\right) E\left(X_{2} / \mu\right)\right)-\mu_{0}^{2} \\
& =E\left(\mu^{2}\right)-\mu_{0}^{2} \\
& =\sigma_{0}^{2}
\end{aligned}
$$

Prova do resultado 3:

$$
\begin{aligned}
V\left(X_{1}\right) & =E\left(V\left(X_{1} / \mu\right)\right)+V\left(E\left(X_{1} / \mu\right)\right) \\
& =E\left(\sigma^{2}\right)+V(\mu) \\
& =\sigma^{2}+\sigma_{0}^{2} .
\end{aligned}
$$


Do Teorema 1.1, sabemos que:

$$
\begin{aligned}
& \hat{E}(\mu / X)=\alpha_{0}+\alpha_{1} X, \\
& \hat{V}(\mu / X)=\sigma_{0}^{2}\left(1-\rho^{2}\right),
\end{aligned}
$$

onde

$$
\begin{aligned}
& \alpha_{0}=\frac{E(\mu) E\left(X^{2}\right)-E(X) E(X \mu)}{V(X)}=\frac{\frac{\mu_{0}}{\sigma_{0}^{2}}}{\frac{1}{\sigma^{2}}+\frac{1}{\sigma_{0}^{2}}}, \\
& \alpha_{1}=\frac{\operatorname{Cov}(X, \mu)}{V(X)}=\frac{\frac{1}{\sigma^{2}}}{\frac{1}{\sigma^{2}}+\frac{1}{\sigma_{0}^{2}}}, \\
& V(\mu / X)=\frac{1}{\frac{1}{\sigma^{2}}+\frac{1}{\sigma_{0}^{2}}} .
\end{aligned}
$$

Assim, temos:

$$
\begin{aligned}
\left(\frac{1}{\sigma^{2}}+\frac{1}{\sigma_{0}^{2}}\right) \hat{E}(\mu / X) & =\frac{X}{\sigma^{2}}+\frac{\mu_{0}}{\sigma_{0}^{2}} \\
\hat{V}^{-1}(\mu / X) & =\frac{1}{\sigma^{2}}+\frac{1}{\sigma_{0}^{2}} .
\end{aligned}
$$

Para uma "futura" observação $X_{2}$ aplicamos o Teorema de Bayes para determinar a Variância $\hat{V}\left(\mu / X_{1}, X_{2}\right)$ e a Esperança $\hat{E}\left(\mu / X_{1}, X_{2}\right)$ Lineares, iterativamente. Para utilizarmos o Teorema de Bayes necessitamos determinar

$$
\hat{E}\left(X_{2} / \mu, X_{1}\right)=\beta_{0}+\beta_{1} \mu+\beta_{2} X_{1} .
$$

Através dos resultados do Teorema 1.4 calculamos

$$
\begin{aligned}
\beta_{0} & =\mu_{0}-\left[\sigma_{0}^{2}, \sigma_{0}^{2}\right] \frac{1}{\sigma^{2} \sigma_{0}^{2}}\left[\begin{array}{cc}
\sigma^{2}+\sigma_{0}^{2} & -\sigma_{0}^{2} \\
-\sigma_{0}^{2} & \sigma_{0}^{2}
\end{array}\right]\left[\begin{array}{l}
\mu_{0} \\
\mu_{0}
\end{array}\right] \\
& =\mu_{0}-\frac{\sigma_{0}^{2}\left(\sigma^{2}+\sigma_{0}^{2}\right)-\sigma_{0}^{2} \sigma_{0}^{2}}{\sigma^{2} \sigma_{0}^{2}} \mu_{0}-\frac{-\sigma_{0}^{2} \sigma_{0}^{2}+\sigma_{0}^{2} \sigma_{0}^{2}}{\sigma^{2} \sigma_{0}^{2}} \mu \\
& =\mu_{0}-\mu_{0}=0,
\end{aligned}
$$




$$
\begin{aligned}
{\left[\beta_{1}, \beta_{2}\right] } & =\left[\sigma_{0}^{2}, \sigma_{0}^{2}\right] \frac{1}{\sigma^{2} \sigma_{0}^{2}}\left[\begin{array}{cc}
\sigma^{2}+\sigma_{0}^{2} & -\sigma_{0}^{2} \\
-\sigma_{0}^{2} & \sigma_{0}^{2}
\end{array}\right] \\
& =\left[\frac{\sigma_{0}^{2}\left(\sigma^{2}+\sigma_{0}^{2}\right)-\sigma_{0}^{2} \sigma_{0}^{2}}{\sigma^{2} \sigma_{0}^{2}}, \frac{-\sigma_{0}^{2} \sigma_{0}^{2}+\sigma_{0}^{2} \sigma_{0}^{2}}{\sigma^{2} \sigma_{0}^{2}}\right] \\
& =[1,0] .
\end{aligned}
$$

Portanto, $\hat{E}\left(X_{1} / \mu, X_{1}\right)=\mu$. Do Teorema de Bayes para Variância e Esperança Lineares para uma "futura" observação $X_{2}$, temos:

$$
\begin{aligned}
\hat{V}^{-1}\left(\mu / X_{1}, X_{2}\right) & =\frac{1}{\sigma^{2}}+\frac{1}{\hat{V}\left(\mu / X_{1}\right)} \\
& =\frac{1}{\sigma^{2}}+\frac{1}{\frac{\sigma^{2} \sigma_{0}^{2}}{\sigma^{2}+\sigma_{0}^{2}}} \\
& =\frac{1}{\sigma_{0}^{2}}+\frac{2}{\sigma^{2}}, \\
\left(\frac{1}{\sigma_{0}^{2}}+\frac{2}{\sigma^{2}}\right) \hat{E}\left(\mu / X_{1}, X_{2}\right) & =\frac{X_{1}}{\sigma^{2}}+\frac{X_{2}}{\sigma^{2}}+\frac{\mu_{0}}{\sigma_{0}^{2}} \\
& =\frac{X_{1}+X_{2}}{\sigma^{2}}+\frac{\mu_{0}}{\sigma_{0}^{2}} .
\end{aligned}
$$

Exemplo 2.2 Então, generalizando o exemplo 2.1, sejam $X_{1}, X_{2}, \ldots, X_{n}, \mu$ variáveis aleatórias condicionalmente independentes e identicamente distribuídas, com $i=$ $1, \ldots, n$. Temos que:

$$
\begin{aligned}
& E(\mu)=\mu_{0}, \\
& V(\mu)=E\left(\mu-\mu_{0}\right)^{2}=\sigma_{0}^{2}, \\
& E\left(X_{i}\right)=E\left(X_{i} / \mu\right)=\mu_{0}, \\
& V\left(X_{i} / \mu\right)=E(X-\mu)^{2}=\sigma^{2},
\end{aligned}
$$

Das hipóteses acima, temos os seguintes resultados: 
1. $\operatorname{Cov}\left(X_{i}, \mu\right)=\sigma_{0}^{2}$,

2. $\operatorname{Cov}\left(X_{i}, X_{2}\right)=\sigma_{0}^{2}$,

3. $V\left(X_{i}\right)=\sigma^{2}+\sigma_{0}^{2}$,

para $i=1, \ldots, n$.

Como Hartigan(1969) apresentou, temos:

$$
\begin{aligned}
& \hat{V}^{-1}\left(\mu / X_{1}, \ldots, X_{n}\right)=\frac{n}{\sigma^{2}}+\frac{1}{\sigma_{0}^{2}}, \\
& \left(\frac{n}{\sigma^{2}}+\frac{1}{\sigma_{0}^{2}}\right) \hat{E}\left(\mu / X_{1}, \ldots, X_{n}\right)=\frac{\sum_{i=1}^{n} X_{i}}{\sigma^{2}}+\frac{\mu_{0}}{\sigma_{0}^{2}} .
\end{aligned}
$$

\section{Prova :}

Antes, vamos mostra que $\hat{E}\left(X_{n+1} / \mu, X_{1}, \ldots, X_{n}\right)=\mu$ utilizando o método indutivo e os resultados obtidos no exemplo 2.1. Vimos que $\hat{E}\left(X_{2} / \mu, X_{1}\right)=\mu$ e vamos supor que $\hat{E}\left(X_{n} / \mu, X_{1}, \ldots, X_{n-1}\right)=\mu$. Então:

$$
\hat{E}\left(X_{n+1} / \mu, X_{1}, \ldots, X_{n}\right)=\hat{E}\left(X_{n} / \mu, X_{1}, \ldots, X_{n-1}\right)+\Delta
$$

onde

$$
\begin{aligned}
\Delta & =\hat{E}\left(X_{n+1} / \mu, X_{1}, \ldots, X_{n}\right)-\hat{E}\left(X_{n} / \mu, X_{1}, \ldots, X_{n-1}\right) \\
& =\mu+\gamma_{0}+\sum_{j=1}^{n} \gamma_{j} X_{j}-\mu \\
& =\gamma_{0}+\sum_{j=1}^{n} \gamma_{j} X_{j} \\
& =0 .
\end{aligned}
$$

Também exemplo 2.1 sabemos que a relação 2.4 acima é valida para $n=1$ e $n=2$. Vamos supor que seja validada para $n$, utilizando o Teorema de Bayes e fazendo $X=\left(X_{1}, \ldots, X_{n}\right)$, temos que: 


$$
\begin{aligned}
\left(\frac{1}{\sigma^{2}}+\frac{1}{V(\mu / X)}\right) \hat{E}\left(\mu / X, X_{n+1}\right) & =\frac{X_{n+1}}{\sigma^{2}}+\frac{\hat{E}(\mu / X)}{\hat{V}(\mu / X)} \\
\left(\frac{1}{\sigma^{2}}+\frac{n}{\sigma^{2}}+\frac{1}{\sigma_{0}^{2}}\right) \hat{E}\left(\mu / X, X_{n+1}\right) & =\frac{X_{n+1}}{\sigma^{2}}+\frac{\sum_{i=1}^{n} X_{i}}{\sigma^{2}}+\frac{\mu_{0}}{\sigma_{0}^{2}}, \\
\left(\frac{n+1}{\sigma^{2}}+\frac{1}{\sigma_{0}^{2}}\right) \hat{E}\left(\mu / X, X_{n+1}\right) & =\frac{\sum_{i=1}^{n+1} X_{i}}{\sigma^{2}}+\frac{\mu_{0}}{\sigma_{0}^{2}}
\end{aligned}
$$

Daí, temos que:

$$
\hat{V}^{-1}\left(\mu / X, X_{n+1}\right)=\frac{n+1}{\sigma^{2}}+\frac{1}{\sigma_{0}^{2}} .
$$

Como as expressões 2.3 e 2.4 são váliadas para $n+1$, portanto, por indução são validas para $n$.

\section{Sumário}

"Se a variância da priori $\sigma_{0}^{2}$ é igual a infinito,

$$
\begin{aligned}
\hat{E}\left(\mu / X_{1}, \ldots, X_{n}\right) & =\frac{\sum_{i=1}^{n} X_{i}}{n}, \\
\hat{V}\left(\mu / X_{1}, \ldots, X_{n}\right) & =\frac{\sigma^{2}}{n},
\end{aligned}
$$

são os familiares estimadores da média e da variância da média, respectivamente."

Podemos agora, a partir dos resultados anteriores e observando o fluxograma na Figura 2.1, para uma futura observação ou observações $\left(X_{n+1}\right)$ aplicar o Teorema de Bayes para determinar Variância e Esperânça Lineares iterativamente, da seguinte forma:

$$
\begin{aligned}
\hat{V}^{-1}\left(\mu / X, X_{n+1}\right) & =\frac{1}{\sigma^{2}}+\frac{1}{\hat{V}(\mu / X)}, \\
\left(\frac{1}{\sigma^{2}}+\frac{1}{V(\mu / X)}\right) \hat{E}\left(\mu / X, X_{n+1}\right) & =\frac{X_{n+1}}{\sigma^{2}}+\frac{\hat{E}(\mu / X)}{\hat{V}(\mu / X)},
\end{aligned}
$$

onde $X=\left(X_{1}, \ldots, X_{n}\right)$. Observamos que, utilizamos somente $n, \sigma^{2}$, a esperança e variância a priori, isto é, $\hat{E}\left(\mu / X_{1}, \ldots, X_{n}\right)$ e $\hat{V}\left(\mu / X_{1}, \ldots, X_{n}\right)$ respectivamente. 


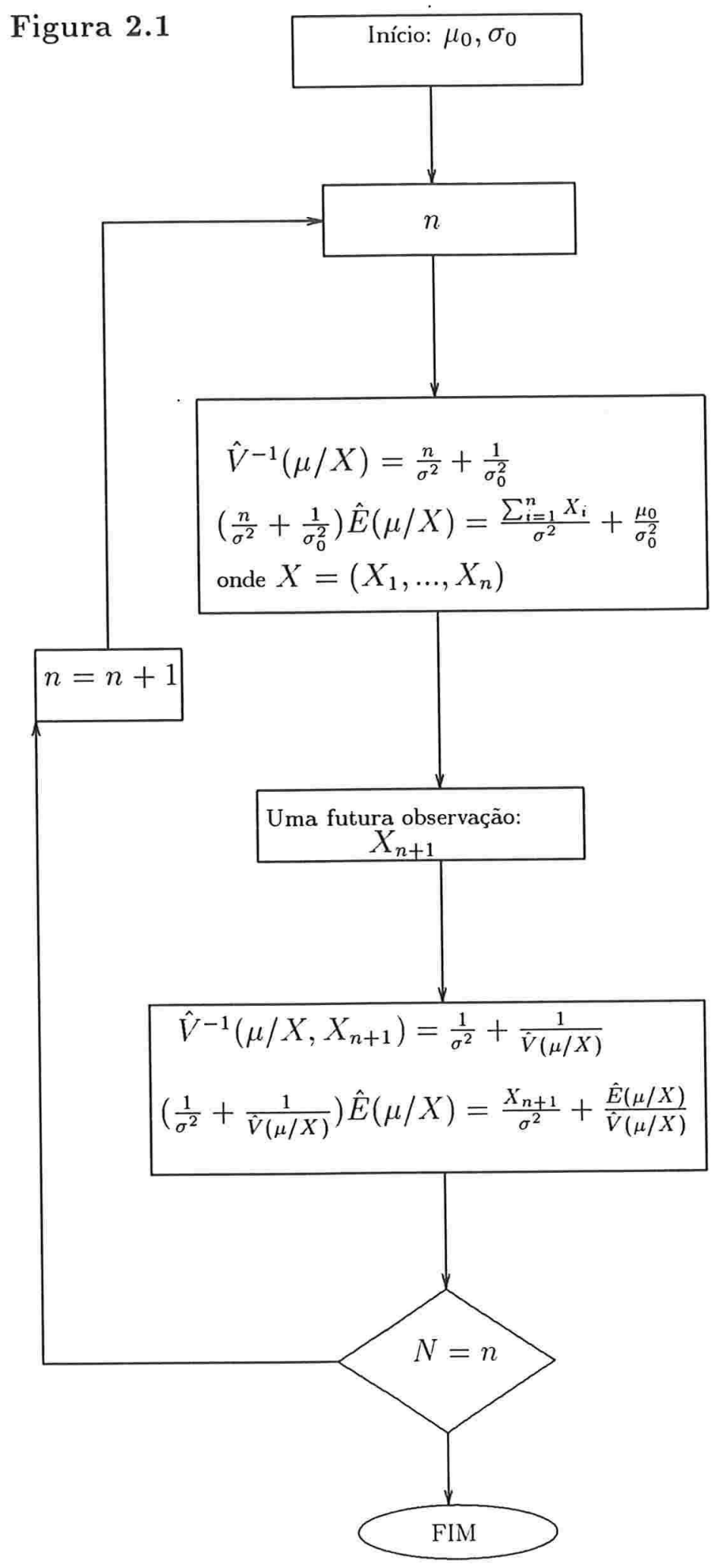

33 
Exemplo 2.3 Neste exemplo, vamos aplicar os resultados obtidos nos exemplos anteriores, no caso onde temos uma variável aleatória com distribuição Binomial. Então, seja $R$ uma variável aleatória binomial com parâmetros $N$ (número de provas) e $p$ (probabilidade de sucesso em cada prova). Sejam $\hat{E}(p)=E(p)=p_{0}$ e $\hat{V}(p)=E\left(p-p_{0}\right)^{2}=v_{0}$, denotaremos:

$$
\begin{aligned}
\hat{E}(R / p) & =\hat{E}(N p)=N \hat{E}(p)=N p_{0} \\
& e \\
\hat{V}(R / p) & =\hat{E}(N p q)=N \hat{E}[p(1-p)] \\
& =N\left[\hat{E}(p)-\hat{E}\left(p^{2}\right)\right]=N\left[p_{0}-p_{0}^{2}-v_{0}\right] \\
& =N\left(p_{0} q_{0}-v_{0}\right) .
\end{aligned}
$$

Portanto, aplicando o Teorema de Bayes para Esperança Linear, Teorema 2.2, como $\hat{E}(R / p)=N p_{0}$ isso implica que $c=N$ e $d=0$, temos:

$$
\begin{aligned}
\hat{V}^{-1}(p / R) & =\frac{N^{2}}{N\left(p_{0} q_{0}-v_{0}\right)}+\frac{1}{v_{0}} \\
& =\frac{N}{p_{0} q_{0}-v_{0}}+\frac{1}{v_{0}}, \\
\hat{V}^{-1}(p / R) \hat{E}(p / R) & =\frac{N R}{N\left(p_{0} q_{0}-v_{0}\right)}+\frac{p_{0}}{v_{0}} \\
& =\frac{R}{p_{0} q_{0}-v_{0}}+\frac{p_{0}}{v_{0}} .
\end{aligned}
$$

Como observou Hartigan(1969), este é o caso onde é perigoso supor uma priori não informativa para $v_{0}$ com um valor muito alto, pois o valor máximo de $v_{0}$ é $p_{0} q_{0}$. 
Escrevendo,

$$
\begin{aligned}
\hat{V}(p / R) & =\frac{v_{0}\left(p_{0} q_{0}-v_{0}\right)}{v_{0} N+p_{0} q_{0}-v_{0}} \\
& e \\
\hat{E}(p / R) & =\frac{v_{0} R}{v_{0} N+p_{0} q_{0}-v_{0}}+\frac{v_{0}\left(p_{0} q_{0}-v_{0}\right)}{v_{0} N+p_{0} q_{0}-v_{0}} \frac{p_{0}}{v_{0}},
\end{aligned}
$$

verificamos que para $v_{0}=p_{0} q_{0}$, temos que a esperança linear $\hat{E}(p / R)=\frac{R}{N}$ é o usual estimador de $p$ e $\hat{V}(p / R)=0$ que é um resultado enganoso, a razão para irregularidade é que fixando $v_{0}=p_{0} q_{0}$ aproxima $p$ para $0(z e r o)$ ou $1 \mathrm{com}$ probabilidade 1 . Como o valor de $p$ será determinado por uma única observação, Hartigan(1969), sugere o valor de $v_{0}$ ser igual a $\frac{p_{0} q_{0}}{2}$ o qual é ponderado por $p_{0}$ dado por uma bservação. Quando $N \rightarrow \infty$ e para $v_{0}<p_{0} q_{0}$, a esperança linear $\hat{E}(p / R)$ difere desprezivelmente do estimador $\frac{R}{N}$, consequentemente o efeito da priori do parâmetros $p_{0}$ e $q_{0}$ na esperança linear diminui. A mesma coisa não pode ser dita sobre a variância linear

$$
\hat{V}(p / R)=\frac{v_{0}\left(p_{0} q_{0}-v_{0}\right)}{v_{0} N+p_{0} q_{0}-v_{0}}
$$

a qual depende criticamente dos parâmetros das prioris.

$\mathrm{O}$ valor de $v_{0}$ é uma medida numérica de incerteza de $p_{0}$, considerando o que já foi afirmado acima, podemos observar que há uma necessidade de o pesquisador ser coerente, isto é, deve remover a "parametrização" arbitrária (Wechsler, 1993), quando emitir sua opinião sobre $v_{0}$, caso contrário, chegará a uma situação absurda onde $\hat{V}(p / R)=0$.

\subsection{Método Linear Bayesiano Multivariado}

Definimos

$$
\hat{E}\left(Y / X_{1}, \ldots, X_{n}\right)=\hat{E}\left(Y / X_{11}, \ldots, X_{1 m_{1}}, X_{21}, \ldots, X_{2 m_{2}}, \ldots, X_{n_{1}}, \ldots, X_{n m_{n}}\right)
$$

onde $\left\{X_{i 1}, \ldots, X_{i m_{i}}\right\}$ são variaveis aletórias componentes de $X_{i}$. Definimos, também,

$$
\hat{Y}=\hat{E}\left(Y / X_{1}, \ldots, X_{n}\right)
$$

para i-ésima componente

$$
\hat{Y}_{i}=\hat{E}\left(Y_{i} / X_{1}, \ldots, X_{n}\right)
$$


Seja $\hat{V}\left(Y / X_{1}, \ldots, X_{n}\right)$ igual à matriz $\left.E[Y-\hat{Y})(Y-\hat{Y})^{\prime}\right]$. A generalização do Teorema de Bayes para Esperança Lienar ( Teorema 2.2)e Variância Linear supondo que exita a priori para os dados $X$, dado uma nova informação $Z$, é representada por:

$$
\begin{aligned}
\hat{V}^{-1}(Y / Z, X) & =C^{\prime} \hat{V}^{-1}(Z / X, Y) C+\hat{V}^{-1}(Y / X) \\
\hat{V}^{-1}(Y / Z, X) \hat{E}(Y / Z, X) & =C^{\prime} \hat{V}^{-1}(Z / X, Y)(Z-D)+\hat{V}^{-1}(Y / X) \hat{E}(Y / X)
\end{aligned}
$$

Os conceitos de esperança linear e variância, viabilizam a aplicação da versão linear do Teorema de Bayes para o caso multivariado, combinando

$$
\begin{gathered}
\hat{E}(Y / X) \text { e } \hat{V}^{-1}(Y / X)(\text { priori }), \\
\hat{E}(Z / Y, X)=C Y+D \text { e } C^{\prime} \hat{V}^{-1}(Z / Y, X) C(\text { dados presentes }), \\
\hat{E}(Y / X, Z) \text { e } \hat{V}^{-1}(Y / X, Z)(\text { posteriori }) .
\end{gathered}
$$

Os resultados acima podem ser provados, como no caso univariado, usando o Teorema de Bayes sobre a Normal multivariada e utilizando o primeiro e o segundo momentos dos vetores aleatórios $X, Y$ e $Z$, considerando que as matrizes $\hat{V}(Y / X), \hat{V}(Z / X, Y)$ e $\hat{V}(Y / Z, X)$ são não-singulares.

\subsection{Regressão}

Uma generalização multivariada do modelo tomando as observações $X_{1}, X_{2}, \ldots, X_{n}$ com:

$$
\hat{E}\left(X_{i} / \mu\right)=\sum a_{i j} \mu_{j} .
$$

As variáveis aleatórias $X_{1}, \ldots, X_{n}$ são não correlacionadas, e cada uma tem variância igual a $\sigma^{2}$.

Com respeito a $\mu$,uma variável aleatória, com

$$
\begin{aligned}
& \hat{E}(\mu)=E(\mu)=\mu_{0}, \\
& \hat{V}(\mu)=E\left[\left(\mu-\mu_{0}\right)\left(\mu-\mu_{0}\right)^{\prime}\right]=\Sigma_{0} .
\end{aligned}
$$


Nós temos que

$$
\begin{aligned}
\hat{V}^{-1}(\mu / X) & =\frac{A^{\prime} A}{\sigma^{2}}+\Sigma_{0}^{-1} \\
\hat{V}^{-1}(\mu / X) \hat{E}(\mu / X) & =\frac{A^{\prime} X}{\sigma^{2}}+\Sigma_{0}^{-1} \mu_{0},
\end{aligned}
$$

pela imediata aplicação da versão Linear do Teorema de Bayes.

Com uma única observação $Z \operatorname{com} \hat{E}(Z / \mu)=\sum a_{j} \mu_{j}$, é incorporado como segue:

$$
\begin{aligned}
\hat{V}^{-1}(\mu / X, Z) & =\frac{A A^{\prime}}{\sigma^{2}}+V^{-1}(\mu / X), \\
\hat{V}^{-1}(\mu / X, Z) \hat{E}(\mu / X, Z) & =\frac{A Z}{\sigma^{2}}+\hat{V}^{-1}(\mu / X) \hat{E}(\mu / X) .
\end{aligned}
$$

Estas equações podem ser usadas como um algoritmo de cálculo para

$$
V^{-1}(\mu / X) \text { e } V^{-1}(\mu / X) \hat{E}(\mu / X)
$$




\section{Capítulo 3}

\section{Caracterização do Filtro de Kalman através do Método Linear Bayesiano}

\subsection{Introdução}

Um processo linear dinâmico produzido por um algoritmo formado por equações de recorrência, originalmente obtidas por Kalman(1960) e Kalman e Bucy(1961), que a partir do conhecimento de observações passadas pode fornecer previsões futuras, é conhecido na literatura como Filtro de Kalman. A simplicidade metodológica do Filtro de Kalman viabiliza sua aplicação em diversas áreas, assim como é uma técnica de grande intresse para a estatística e de grande utilidade em modelos de regressão lineares e análise de séries temporais que, neste caso, tem como hipótese básica a existência de componentes não-observáveis. Este algoritmo, Filtro de Kalman, foi construído pela primeira vez no contexto bayesiano por Harrison e Stevens(1976) com o objetivo de identifição e implementação.

Como foi observado por Meinhold e Singpurwalla (1983), o Filtro de Kalman pode ser facilmente compreendido se é tratado como um problema de infêrencia Bayesiana e empregando resultados bem conhecidos e elementares da estatística multivariada. Analisaremos a seguir o modelo de Filtro de Kalman considerando os argumentos propostos por Meinhold e Singpurwalla (1983) e caraterizaremos através do Método Linear Bayesiano. 


\subsection{Modelo de Filtro de Kalman}

Apresentaremos a definição de Filtro de Kalman proposta por Meinhold e Singpurwalla(1983). Sejam $Y_{t}, Y_{t-1}, \ldots, Y_{1}$ (os quais podem ser vetores ou escalares), denotaremos a variável de interesse o tempo $t, t-1, \ldots, 1$. Assumimos que $Y_{t}$ depende de uma quantidade não-observável $\theta_{t}$, conhecido como estado da natureza. O objetivo é fazer inferência sobre $\theta_{t}$, o qual pode ser vetor ou escalar, com dimensão independente da dimensão de $Y_{t}$. A relação entre $Y_{t}$ e $\theta_{t}$ é linear especificada por

$$
Y_{t}=F_{t} \theta_{t}+v_{t}
$$

onde $F_{t}$ é uma quantidade conhecida no tempo $t$. É assumido que os erros $v_{t}$ são normalmente distribuídos com média zero e variância conhecida $V_{t}=E\left(v_{t}, v_{t}^{\prime}\right)$, representada por $v_{t} \sim N\left(0, V_{t}\right)$.

A principal diferença entre o Filtro de Kalman e o modelo linear convencional é que o estado da natureza, representado pelo coeficiente de regressão que é assumido constante no modelo linear, pode mudar, ou melhor, é uma função do tempo $t$ no Filtro de Kalman. Esta característica dinâmica do Filtro de Kalman é incorporada via o sistema de equações,

$$
\theta_{t}=G_{t} \theta_{t-1}+w_{t}
$$

onde $G_{t}$ é uma quantidade conhecida no sistema de equações e o erro $w_{t}$ é normalmente distribuída com média zero e variância conhecida $W_{t}=E\left(w_{t}, w_{t}^{\prime}\right)$, representada por $w_{t} \sim N\left(0, W_{t}\right)$. O estado da natureza $\theta_{t}$ muda com o tempo em vários sistemas físicos, mas $F_{t}$ e $G_{t}$ que especificam a equação observacional 3.1 e sistema de equações 3.2 , respectivamente, podem, ou não, mudar com o tempo, assim como as variâncias $V_{t}$ e $W_{t}$.

\subsection{Processo Iterativo de Estimação}

Como já foi afirmado acima, o objetivo do Filtro de Kalman é construir inferência sobre o estado da natureza $\theta_{t}$ através de um procedimento recursivo. Como argumentaram Meinhold e Singpurwalla (1983), a idéia básica é que dada as observações $Y_{t}=\left(Y_{t}, \ldots, Y_{1}\right)$, a inferência sobre $\theta_{t}$, pode ser completada através da aplicação direta 
do Teorema de Bayes:

$$
P\left(\theta_{t} / Y_{t}\right) \propto P\left(Y_{t} / \theta_{t}, Y_{t-1}\right) * P\left(\theta_{t} / Y_{t-1}\right),
$$

onde $P\left(\theta_{t} / Y_{t}\right)$ representa a distribuição a posteriori do estado da natureza $\theta_{t}$ dada as observações $Y_{t}=\left(Y_{t}, \ldots, Y_{1}\right), P\left(Y_{t} / \theta_{t}, Y_{t-1}\right)$ é a verossimilhança e $P\left(\theta / Y_{t-1}\right)$ a priori de $\theta_{t}$ dado $Y_{t-1}$.

O procedimento iterativo pode ser melhor explicado se nossa atenção estiver voltada para o tempo $t-1, t=1,2, \ldots$; e observarmos os dados, $Y_{t-1}=\left(Y_{t-1}, Y_{t-2}, \ldots, Y_{1}\right)$, utilizando a álgebra de matrizes e admitindo que $Y$ e/ou $\theta$ são vetores, sem explicitar como tal. Para $t-1$, nosso estado de conhecimento sobre $\theta_{t-1}$ é representado pela distribuição

$$
\theta_{t-1} / Y_{t-1} \sim N\left(\hat{\theta}_{t-1}, \Sigma_{t-1}\right)
$$

onde $\hat{\theta}_{t-1}$ e $\Sigma_{t-1}$ são esperança e variância da distribuiçao de $\theta_{t-1} / Y_{t-1}$. É interessante observar que o procedimento recursivo é iniciado no tempo $t=0 \operatorname{com} \hat{\theta}_{0}$ e $\Sigma_{0}$ sendo a média e a variância de $\theta_{0}$ respectivamente, que representam a nossa melhor avaliação no estágio inicial de conhecimento do processo.

Nós vamos avaliar nosso conhecimento no tempo $t$ observando os dois estágios seguintes:

1. A priori para a observação $Y_{t}$.

2. Resultado obtido após ter observado $Y_{t}$.

No estágio 1, a priori para a observação $Y_{t}$, nossa melhor escolha para $\theta_{t}$ é controlada pelo sistema de equações 3.2 que é dada por

$$
\theta_{t}=G_{t} \theta_{t-1}+w_{t},
$$

onde $\theta_{t-1}$ é descrito por 3.4 , nosso conhecimento sobre $\theta_{t}$ é incorporado na distribuição de probabilidade

$$
\theta_{t} / Y_{t-1} \sim N\left(G_{t} \hat{\theta}_{t-1}, R_{t}=G_{t} \Sigma_{t-1} G_{t}^{\prime}+W_{t}\right)
$$

que é nossa distribuição a priori.

No estágio 2, nossa meta é calcular a posteriori de $\theta_{t}$ através de 3.2 dada a observação $Y_{t}$. Primeiro necessitamos conhecer a verossimilhança $\mathcal{L}\left(Y_{t} / \theta_{t}\right)$, ou melhor, $P\left(Y_{t} / \theta_{t}, Y_{t-1}\right)$, como apresentaram Meinhold e Singpurwalla (1983) com os argumentos que se seguem. 
O erro de previsão de $Y_{t}$ no ponto $t-1$, representado por $e_{t}$ e definido por

$$
\begin{aligned}
\epsilon_{t} & =Y_{t}-\hat{Y}_{t} \\
& =Y_{t}-E\left(Y_{t}\right) \\
& =Y_{t}-F_{t} G_{t} \hat{\theta}_{t-1} .
\end{aligned}
$$

Como $F_{t}, G_{t}$ e $\hat{\theta}_{t-1}$ são todos conhecidos por hipótese, observar $Y_{t}$ é equivalente observar $e_{t}$. Então, por 3.3 podemos escrever

$$
\begin{aligned}
P\left(\theta_{t} / Y_{t}, Y_{t-1}\right) & =P\left(\theta / e_{t}, Y_{t-1}\right) \\
& \propto P\left(e_{t} / \theta, Y_{t-1}\right) * P\left(\theta / Y_{t-1}\right),
\end{aligned}
$$

onde $P\left(e_{t} / \theta_{t}, Y_{t-1}\right)$ é verossimilhança e $P\left(\theta_{t} / Y_{t-1}\right)$ é a priori. Para

$$
\begin{aligned}
& Y_{t}=F_{t} \theta_{t}+v_{t} \\
& e_{t}=Y_{t}-F_{t} G_{t} \hat{\theta}_{t-1} \mathrm{e} \\
& E\left(e_{t} / \theta_{t}, Y_{t-1}\right)=F_{t}\left(\theta_{t}-G_{t} \hat{\theta}_{t-1}\right)
\end{aligned}
$$

como $v_{t} \sim N\left(0, V_{t}\right)$, segue que a verossimilhança é descrita por

$$
e_{t} / \theta_{t}, Y_{t-1} \sim N\left(F_{t}\left(\theta_{t}-G_{t} \hat{\theta}_{t-1}\right), V_{t}\right) \text {. }
$$

Aplicando o Teorema de Bayes obtemos

$$
P\left(\theta_{t} / Y_{t}, Y_{t-1}\right)=\frac{P\left(e_{t} / \theta, Y_{t-1}\right) * P\left(\theta / Y_{t-1}\right)}{\int_{\forall \theta_{t}} P\left(e_{t}, \theta / Y_{t-1}\right) d \theta_{t}} .
$$

Esta é a melhor descrição do nosso conhecimento sobre $\theta_{t}$ no tempo $t$. Uma vez que $P\left(\theta_{t} / Y_{t}, Y_{t-1}\right)$ é calculado, podemos voltar para 3.3 e iniciar um novo ciclo do processo iterativo. Na seção seguinte mostraremos que a distribuição 3.9 é da forma presente em 3.3 .

\subsection{Determinação da Distribuição Posteriori através da Normal}

Para obter $P\left(\theta_{t} / Y_{t}, Y_{t-1}\right)$ vamos utilizar os resultados apresentados no Capítulo 1 , onde analisamos algumas das propriedades da distribuição Normal. Aqui nesta seção, não vamos determinar a posteriori utilizando a relação 3.9. 
Como foi apresentado no Teorema 1.7 e utilizando a notação da álgebra matricial, sejam $X_{1}$ e $X_{2}$ variáveis aleatórias com distribuição conjunta Normal com média $\mu_{1}$ e $\mu_{2}$, respectivamente, e a matriz de covariâncias

$$
\left(\begin{array}{ll}
\Sigma_{11} & \Sigma_{12} \\
\Sigma_{21} & \Sigma_{22}
\end{array}\right)
$$

denotaremos por

$$
\left(\begin{array}{l}
X_{1} \\
X_{2}
\end{array}\right) \sim N\left[\left(\begin{array}{l}
\mu_{1} \\
\mu_{2}
\end{array}\right),\left(\begin{array}{cc}
\Sigma_{11} & \Sigma_{12} \\
\Sigma_{21} & \Sigma_{22}
\end{array}\right)\right] .
$$

Se 3.10 é válida, a distribuição condicional de $X_{1}$ dado $X_{2}$, como foi determinada no Capítulo 1, é da forma

$$
X_{1} / X_{2}=x_{2} \sim N\left(\mu_{1}+\Sigma_{12} \Sigma_{22}^{-1}\left(x_{2}-\mu_{2}\right), \Sigma_{11}-\Sigma_{12} \Sigma_{22}^{-1} \Sigma_{21}\right)
$$

A quantidade $\mu_{1}+\Sigma_{12} \Sigma_{22}^{-1}\left(x_{2}-\mu_{2}\right)$ é chamada de função de regressão, e $\Sigma_{12} \Sigma_{22}^{-1}$ é o coeficiente de mínimos quadrados de regressão de $X_{1}$ dado $X_{2}$. Como podemos verificar, 3.10 implica 3.11. Podemos observar também que se 3.11 é válida e $X_{2} \sim N\left(\mu_{2}, \Sigma_{22}\right)$, então 3.11 será verdadeira. Usaremos esta relação inversa (Meinhold e Singpurwalla, 1983) para determinar a distribuição posteriori. No primeiro momento supriremos a variárivel aleatória $Y_{t-1}$, fazendo $X_{1}$ corresponder a $e_{t}$ e $X_{2}$ a $\theta_{t}$, representaremos estas correspondências por $X_{1} \cong e_{t}$ e $X_{2} \cong \theta_{t}$, respectivamente. Então,

$$
\theta_{t} / Y_{t-1} \sim N\left(G_{t} \hat{\theta}_{t-1}, R_{t}\right)
$$

e desta forma

$$
\begin{aligned}
\mu_{2} & \cong G_{t} \hat{\theta}_{t-1} \\
& e \\
\Sigma_{22} & \cong R_{t}=G_{t} \Sigma_{t-1} G_{t}^{\prime}+w_{t} .
\end{aligned}
$$

Substituindo em $3.11 X_{1}, X_{2}, \mu_{2}$ e $\Sigma_{22}$ por $e_{t}, \theta_{t}, G_{t} \hat{\theta}_{t-1}$ e $R_{t}$, respectivamente, e utilizando o resultado 3.9 temos que

$$
e_{t} / \theta_{t}, Y_{t-1} \sim N\left(F_{t}\left(\theta_{t}-G_{t} \hat{\theta}_{t-1}\right), V_{t}\right)
$$

Então, podemos escrever que

$$
\mu_{1}+\Sigma_{12} R_{t}^{-1}\left(\theta_{t}-G_{t} \hat{\theta}_{t-1}\right) \cong F_{t}\left(\theta_{t}-G_{t} \hat{\theta}_{t-1}\right) .
$$


Escrevemos também que $\mu_{1} \cong 0$ e $\Sigma_{12} \cong F_{t} R_{t}$, pela mesma razão, temos que

$$
\Sigma_{11}-\Sigma_{12} \Sigma_{22}^{-1} \Sigma_{21}=\Sigma_{11}-F_{t} R_{t} F_{t}^{\prime} \cong V_{t}
$$

Portanto, $\Sigma_{11} \cong V_{t}+F_{t} R_{t} F_{t}^{\prime}$.

Com os argumentos acima mencionados, concluimos que a distribuição conjunta de $\theta_{t}$ e $e_{t}$, dado $Y_{t-1}$, é descrita por

$$
\left[\left(\begin{array}{c}
\theta_{t} \\
e_{t}
\end{array}\right) / Y_{t-1}\right] \sim N\left[\left(\begin{array}{c}
G_{t} \hat{\theta}_{t-1} \\
0
\end{array}\right),\left(\begin{array}{cc}
R_{t} & R_{t} F_{t}^{\prime} \\
F_{t} R_{t} & V_{t}+F_{t} R_{t} F_{t}^{\prime}
\end{array}\right)\right] .
$$

Condicionando em $e_{t}$ e $Y_{t}$, fazendo 3.13 corresponder a 3.10 obtemos então, via 3.11 o resultado

$$
\theta_{t} / e_{t}, Y_{t-1} \sim N\left[G_{t} \hat{\theta}_{t-1}+R_{t} F_{t}^{\prime}\left(V_{t}+F_{t} R_{t} F_{t}^{\prime}\right)^{-1} e_{t}, R_{t}-R_{t} F_{t}^{\prime}\left(V_{t}+F_{t} R_{t} F_{t}^{\prime}\right)^{-1} F_{t} R_{t}\right]
$$

Resumindo, depois do tempo $t-1$, temos uma distribuição a posteriori para $\theta_{t-1}$ com média $\hat{\theta}_{t-1}$ e variância $\Sigma_{t-1}$ em 3.4. Criamos uma priori para $\theta_{t}$ com média $G_{t} \hat{\theta}_{t-1}$ e variância $R_{t}=G_{t} \Sigma_{t-1} G_{t}^{\prime}+W_{t} 3.5$, analisamos a verossimilhança para $e_{t}=$ $Y_{t}-F_{t} G_{t} \hat{\theta}_{t-1}$ em 3.6 , chegamos a posteriori de $\theta_{t}$ que tem média

$$
\hat{\theta}_{t}=G_{t} \hat{\theta}_{t-1}+R_{t} F_{t}^{\prime}\left(V_{t}+F_{t} R_{t} F_{t}^{\prime}\right)^{-1} e_{t}
$$

e variância

$$
\Sigma_{t}=R_{t}-R_{t} F_{t}^{\prime}\left(V_{t}+F_{t} R_{t} F_{t}^{\prime}\right)^{-1} F_{t} R_{t}
$$

Na Figura 3.1 apresentamos um fluxograma onde sumarisamos as idéias de Meinhold e Singpurwalla (1983). 


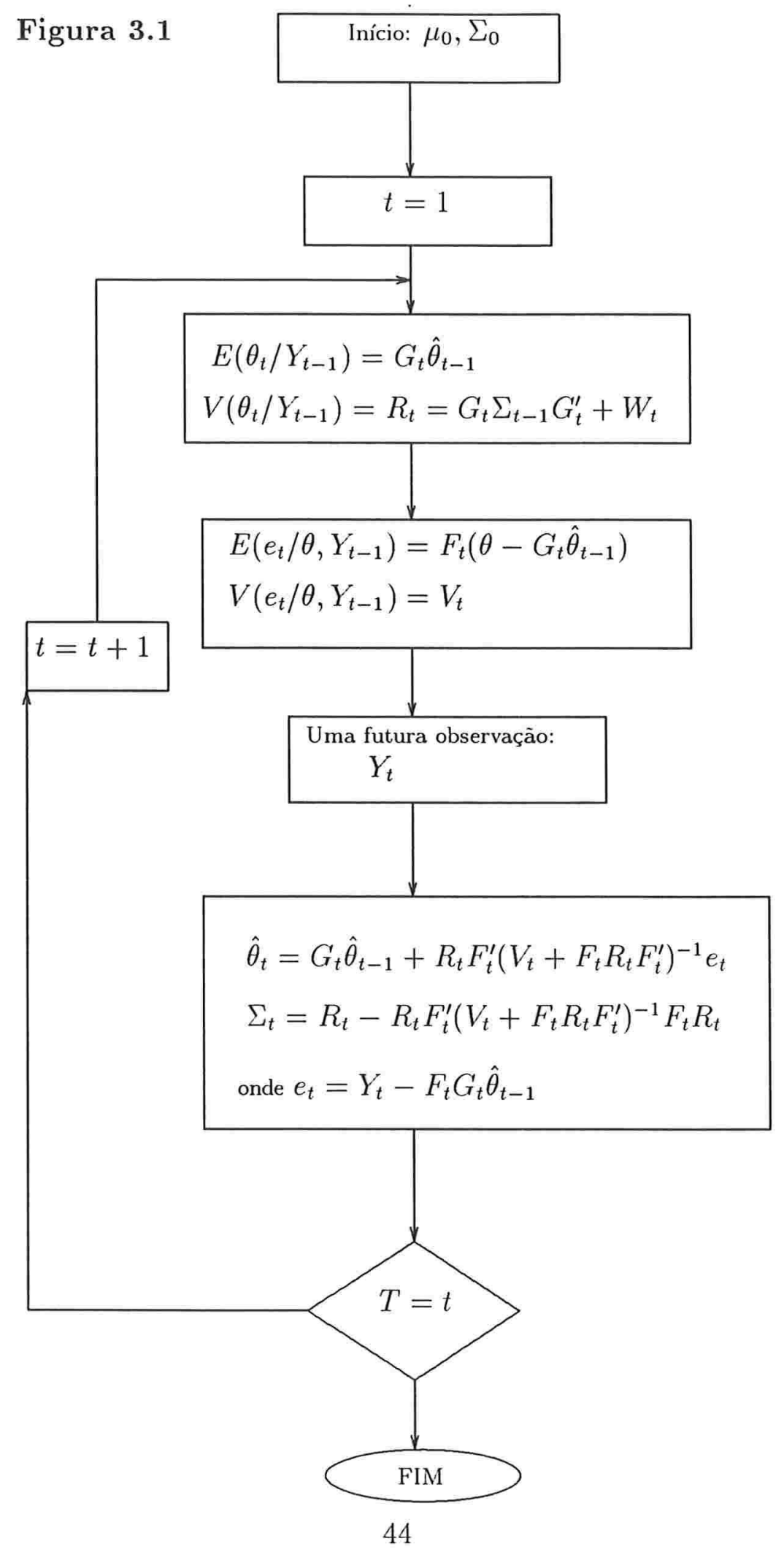




\subsection{Caracterização do Filtro de Kalman através do Método Linear Bayesiano}

Para caracterizar o Filtro de Kalman através do Método Linear Bayesiano, utilizaremos os resultados apresentados nos capítulos anteriores. Como foi visto no Capítulo 2, Hartigan (1969) define esperança linear através dos momentos de primeira e segunda ordem. Então, seja $\left(Y_{t}, \theta_{t}\right)$ um vetor aleatório em $\mathbb{R}^{2}$, a esperança linear de $\theta_{t}$ dado $Y_{t}$ é da forma

$$
\hat{E}\left(\theta_{t} / Y_{t}=y_{t}\right)=\alpha_{0}+\alpha_{1} y_{t}
$$

onde

$$
\begin{aligned}
\alpha_{0} & =\frac{E\left(\theta_{t}\right) E\left(Y_{t}^{2}\right)-E\left(Y_{t}\right) E\left(\theta_{t} Y_{t}\right)}{\operatorname{Var}\left(Y_{t}\right)} \\
e & \\
\alpha_{1} & =\frac{E\left(\theta_{t} Y_{t}\right)-E\left(\theta_{t}\right) E\left(Y_{t}\right)}{\operatorname{Var}\left(Y_{t}\right)},
\end{aligned}
$$

como foi determinado no Teorema 1.1 e a variância linear ( Capítulo 2) é

$$
\hat{V}\left(\theta_{t} / Y_{t}\right)=S\left(\alpha_{0}, \alpha_{1}\right)
$$

Teorema 3.1 Considerando os argumentos de Meinhold e Singpurwalla (1983) e Hartigan (1969), seja $\left(Y_{t}, \theta_{t}\right)$ um vetor aleatório em $\mathbb{R}^{2}$ não-singular e com momentos de primeira e segunda ordem finitos. Então, a esperança linear de $\theta_{t}$ dado $Y_{t}$, é da forma

$$
\begin{aligned}
\hat{E}\left(\theta_{t} / Y_{t}\right)=\hat{\theta}_{t} & =G_{t} \hat{\theta}_{t-1}-\frac{F_{t} G_{t} \hat{\theta}_{t-1}\left(F_{t} G_{t}^{2} \Sigma_{t-1}+F_{t} W_{t}\right)}{F_{t}^{2} G_{t}^{2} \Sigma_{t-1}+F_{t}^{2} W_{t}+V_{t}} \\
& +\frac{F_{t} G_{t}^{2} \Sigma_{t-1}+F_{t} W_{t}}{F_{t}^{2} G_{t}^{2} \Sigma_{t-1}+F_{t}^{2} W_{t}+V_{t}} Y_{t}
\end{aligned}
$$

Prova: O Filtro de Kalman definido na seção 3.2 é dado por

$$
\left\{\begin{array}{l}
Y_{t}=F_{t} \theta_{t}+v_{t}, v_{t} \sim N\left(0, V_{t}\right) \\
\theta_{t}=G_{t} \theta_{t-1}+w_{t}, w_{t} \sim N\left(0, W_{t}\right)
\end{array}\right.
$$


Podemos verificar que

$$
\begin{aligned}
E\left(\theta_{t}\right) & =G_{t} \hat{\theta}_{t-1}, \\
E\left(Y_{t}\right) & =F_{t} G_{t} \hat{\theta}_{t-1}, \\
\operatorname{Var}\left(Y_{t}\right) & =F_{t}^{2} G_{t}^{2} \Sigma_{t-1}+F_{t}^{2} W_{t}+V_{t}, \\
E\left(Y_{t}^{2}\right) & =F_{t}^{2} G_{t}^{2} \Sigma_{t-1}+F_{t}^{2} W_{t}+V_{t}+\left(F_{t} G_{t} \hat{\theta}_{t-1}\right)^{2} \\
E\left(\theta_{t} Y_{t}\right) & =F_{t} G_{t}^{2} \Sigma_{t-1}+F_{t} G_{t}^{2} \hat{\theta}_{t-1}^{2}+F_{t} W_{t}
\end{aligned}
$$

e substituindo estes resultados na equação 3.17 o teorema está provado. De maneira análoga, podemos verificar que a variância linear é da forma

$$
\hat{V}\left(\theta_{t} / Y_{t}\right)=\Sigma_{t}=G_{t}^{2} \Sigma_{t-1}+W_{t}-\frac{\left(F_{t} G_{t}^{2} \Sigma_{t-1}+F_{t} W_{t}\right)^{2}}{F_{t}^{2} G_{t}^{2} \Sigma_{t-1}+F_{t}^{2} W_{t}+V_{t}} .
$$

Traduzindo os resultados acima para a notação da álgebra matricial, temos

$$
\begin{aligned}
\hat{E}\left(\theta_{t} / Y_{t}\right)=\hat{\theta}_{t} & =G_{t} \hat{\theta}_{t-1}+\left(G_{t} \Sigma_{t-1} G_{t}^{\prime}+W_{t}\right) * \\
& * F_{t}^{\prime}\left(V_{t}+F_{t}\left(G_{t} \Sigma_{t-1} G_{t}^{\prime}+W_{t}\right) F_{t}^{\prime}\right)^{-1}\left(Y_{t}-F_{t} G_{t} \hat{\theta}_{t-1}\right),
\end{aligned}
$$

onde

$$
\begin{aligned}
R_{t} & =G_{t} \Sigma_{t} G_{t}^{\prime}+W_{t} \\
& e \\
e_{t} & =Y_{t}-F_{t} G_{t} \hat{\theta}_{t-1},
\end{aligned}
$$

$\log 0$

$$
\hat{E}\left(\theta_{t} / Y_{t}\right)=\hat{\theta}_{t}=G_{t} \hat{\theta}_{t-1}+R_{t} F_{t}^{\prime}\left(V_{t}+F_{t} R_{t} F_{t}^{\prime}\right)^{-1} e_{t} .
$$

Utilizando os mesmos argumentos obtemos

$$
\hat{V}\left(\hat{\theta}_{t} / Y_{t}\right)=\Sigma_{t}=R_{t}-R_{t} F_{t}^{\prime}\left(V_{t}+F_{t} R_{t} F_{t}^{\prime}\right)^{-1} F_{t} R_{t} .
$$

Estes resultados são os mesmos obtidos por Meinhold e Singpurwalla (1983). Desta forma, construímos a Caracterização do Filtro de Kalman através do Método Linear Bayesiano como Hartigan(1969) definiu. 


\section{Sumário do Teorema 3.1}

A formulação do Filtro de Kalman apresentada utilizando um argumento bayesiano e as propriedades da distribuição Normal multivariada, proporciona uma fácil caracterização do Filtro de Kalman através da Esperança Linear, considerando que

$$
\begin{aligned}
E\left(\theta_{t} / Y_{t-1}\right) & =G_{t} \hat{\theta}_{t-1}, \\
V\left(\theta_{t} / Y_{t-1}\right) & =G_{t}^{2} \Sigma_{t-1}+W_{t}, \\
V\left(e_{t} / \theta_{t}, Y_{t-1}\right) & =V\left(Y_{t} / \theta, Y_{t-1}\right)=V_{t},
\end{aligned}
$$

desta forma, com estes resultados acima, obtidos nas fórmulas 3.5 e 3.8 respectivamente, podemos escrever o Filtro de Kalman exatamente como no Teorema de Bayes para a Esperança Linear, levando em conta que o Teorema 1.7 estabeleceu a coincidência entre esperança condicionais e retas de regressão lineares no caso de normalidade da distribuiçao conjunta, que é o caso do Filtro de Kalman; portanto, temos que

$$
\begin{aligned}
\hat{V}^{-1}\left(\theta_{t} / Y_{t}\right) & =\frac{F_{t}^{2}}{\hat{V}\left(Y_{t} / \theta_{t}, Y_{t-1}\right)}+\frac{1}{\hat{V}\left(Y_{t} / \theta_{t}\right)}, \\
\hat{V}^{-1}\left(\theta_{t} / Y_{t}\right) \hat{E}\left(\theta_{t} / Y_{t}\right) & =\frac{\hat{E}\left(\theta_{t} / Y_{t-1}\right)}{\hat{V}\left(\theta_{t} / Y_{t-1}\right)}+\frac{F_{t}}{\hat{V}\left(Y_{t} / \theta_{t}, Y_{t-1}\right)} Y_{t} .
\end{aligned}
$$

Podemos ver também, que os conceitos de esperança linear e variância, viabilizam a aplicação da versão linear do Teorema de Bayes no Filtro de Kalman, combinando

$$
\begin{gathered}
\hat{E}\left(\theta_{t} / Y_{t-1}\right) \text { e } \hat{V}\left(\theta_{t} / Y_{t-1}\right)(\text { priori }), \\
\hat{E}^{-1}\left(Y_{t} / \theta, Y_{t-1}\right) e \hat{V}^{-1}\left(Y_{t} / \theta, Y_{t-1}\right) F_{t}^{2}(\text { dados presentes }), \\
\hat{E}\left(\theta_{t} / Y_{t}\right) e \hat{V}^{-1}\left(\theta_{t} / Y_{t}\right)(\text { posterior } i) .
\end{gathered}
$$

Com os resultados do Teorema 3.1, detalhados acima, onde verificamos que $d=0$ e $c=F_{t}$, podemos concluir que o Filtro de Kalman é um caso patricular do Método Linear Bayesiano. 


\subsection{Um Exemplo Numérico}

Este exemplo foi proposto por Meinhold e Singpurwalla (1983). Consideramos o Filtro de Kalman definido por

$$
\left\{\begin{array}{l}
Y_{t}=F_{t} \theta_{t}+v_{t}, v_{t} \sim N\left(0, V_{t}\right) \\
\theta_{t}=G_{t} \theta_{t-1}+w_{t}, w_{t} \sim N\left(0, W_{t}\right)
\end{array}\right.
$$

onde os valores a priori são dados por $\theta_{0}=4.183$ e $\Sigma_{0}=1$. Fixamos, também, $V_{t}=2$ e $W_{t}=1$, e incorporamos o componente cíclico de $\theta_{t}$, através da equação

$$
G_{t}=\frac{1}{2} \operatorname{sen}\left(\frac{\pi}{2}(2 t+1)\right)=\frac{(-1)^{t}}{2} .
$$

Determinamos $F_{t}$ utilizando uma regressão ordinária e supomos, ainda, que $v_{t}$ e $w_{t}$ são gerados através de uma tabela de números aleatórios normalmente distribuídos. Atualizamos os valores de $\theta_{0}=4.183$ e $\Sigma_{0}=1$ através do processo recursivo, representado aqui pela Caracterização do Filtro de Kalman através do Método Linear Bayesiano e calculado por (Teorema 3.1)

$$
\begin{aligned}
\hat{V}^{-1}\left(\theta_{t} / Y_{t}\right) & =\frac{F_{t}^{2}}{\hat{V}\left(Y_{t} / \theta_{t}, Y_{t-1}\right)}+\frac{1}{\hat{V}\left(Y_{t} / \theta_{t}\right)}, \\
\hat{V}^{-1}\left(\theta_{t} / Y_{t}\right) \hat{E}\left(\theta_{t} / Y_{t}\right) & =\frac{\hat{E}\left(\theta_{t} / Y_{t-1}\right)}{\hat{V}\left(\theta_{t} / Y_{t-1}\right)}+\frac{F_{t}}{\hat{V}\left(Y_{t} / \theta_{t}, Y_{t-1}\right)} Y_{t},
\end{aligned}
$$

onde

$$
\begin{aligned}
E\left(\theta_{t} / Y_{t-1}\right) & =G_{t} \hat{\theta}_{t-1}, \\
V\left(\theta_{t} / Y_{t-1}\right) & =G_{t}^{2} \Sigma_{t-1}+W_{t}, \\
V\left(e_{t} / \theta_{t}, Y_{t-1}\right) & =V\left(Y_{t} / \theta, Y_{t-1}\right)=V_{t},
\end{aligned}
$$

Apresentamos na Tabela 3.1 os resultados calculados, como era esperado, estes resultados são iguais aos obtidos por Meinhold e Singpurwalla (1983). 
Tabela 3.1

\begin{tabular}{r|r|r|r|r|r|r|r}
\hline & & & & & & & \\
$t$ & $v_{t}$ & $w_{t}$ & $F_{t}$ & $\theta_{t}$ & $Y_{t}$ & $\hat{\theta}_{t}$ & $\Sigma_{t}$ \\
\hline 0 & & & & -0.353 & & 4.183 & 1 \\
1 & -.376 & .887 & 1.3 & 1.063 & 1.007 & -.619 & .608 \\
2 & .023 & -1.021 & .8 & -.489 & -.368 & -.350 & .842 \\
3 & -.898 & -1.207 & .9 & -.962 & -1.764 & -.527 & .812 \\
4 & 1.645 & .150 & 1.1 & -.331 & 1.281 & .338 & .696 \\
5 & -.701 & -.329 & 1.2 & -.163 & -.897 & -.434 & .636 \\
6 & -.257 & .448 & 1.0 & .366 & .109 & -.097 & .734 \\
7 & -1.766 & .403 & 1.1 & .220 & -1.524 & -.550 & .690 \\
8 & -1.551 & -1.069 & .9 & -.959 & -2.414 & -1.050 & .795 \\
9 & .443 & .186 & .9 & .666 & 1.042 & .732 & .807 \\
10 & .300 & -.267 & 1.0 & .066 & .366 & .366 & .751 \\
11 & -.745 & .406 & 1.2 & .373. & -.297 & -.213 & .640 \\
12 & -1.175 & -.789 & .8 & -.602 & -1.657 & -.638 & .846 \\
13 & 1.281 & .386 & 1.1 & .687 & 2.037 & .967 & .699 \\
14 & -1.017 & -.753 & .7 & -.409 & -1.304 & -.041 & .912 \\
15 & -.991 & -.120 & .9 & .085 & -.915 & -.324 & .820 \\
16 & .861 & .524 & 1.0 & .566 & 1.427 & .436 & .752 \\
17 & -1.047 & .224 & 1.3 & -.059 & -1.124 & -.542 & .593 \\
18 & 076 & -.356 & 1.1 & -.386 & -.348 & -.290 & .678 \\
19 & 2.498 & -.907 & 1.2 & -.714 & 1.641 & .704 & .635 \\
20 & 1.482 & -.881 & .9 & -1.238 & .368 & .370 & .789 \\
21 & -1.683 & .023 & .7 & .642 & -1.234 & -.543 & .926 \\
22 & 1.072 & .632 & .6 & .953 & 1.644 & .275 & 1.008 \\
23 & -.684 & -.314 & 1.1 & -.791 & -1.554 & -.687 & .712 \\
24 & -.580 & -.217 & 1.0 & -.612 & -1.192 & -.658 & 741 \\
25 & -1.894 & 1.927 & .9 & 2.233 & .116 & .264 & .801 \\
\hline
\end{tabular}




\section{Bibliografia}

[1] Aitchison, J. and Dunsmore,L.R.(1975). Statistical Prediction Analysis, Cambridge.:Cambridge University Press.

[2] Araújo, Péricles César de e Wechsler, S. (1996). Método Linear Bayesiano, Resumos, Caxambu, XII SINAPE.

[3] Box, G.E.P and Tiao,G.C. (1973). Bayesian Inference in Statistical Analysis, Mass.: Addison-Wesley.

[4] Gamerman, D. e Migon,H.S.(1993). Inferência Estatistica: Uma abordagem integrada, IM-UFRJ.

[5] Gamerman, Dani (1996). Simulação Estocástica via Cadeias de Markov, Caxanbu, XII SINAPE.

[6] Harrison, P.J., and Stevens, C.F.(1976). Bayesian Forecasting (with discussion),JRSS, Ser.B, 38,205-247.

[7] Hartigan, J.A. (1969). Linear Bayesian Methods, JRSS B, 31, 446-454.

[8] Inoue, Lurdes Y.T. (1995). Desenvolvimento e Implicaçôes do Princípio da Verossimilhança, São Paulo, IME-USP(tese de mestrado).

[9] James, B.R. (1981). Probabilidade: Um Curso em Nivel Intermediário, IMPA, Projeto Euclides, CNPq.

[10] Kagan,A.M. and Karpov, Y.N. (1972). Bayesian formulation of the estimation problem for the location parameter(in Russian), Zapiski Nauchnych Seminarov Leningrad Section of the Mathematical Institute, 29.

[11] Kalman, R.E. (1960). A New Approach to Linear Filtering and Prediction, Trans. ASME, J.Basic Eng. 82D, 1, 35-45. 
[12] Kalman, R.E. and Bucy, R.S.(1961). New Resulte in Linear Filtering and Prediction Theory, Trans. ASME, J.Basic Eng. 82D, 1, 95-108.

[13] Kendall, M. and Stuart, A.(1979). The Advanced Theory of Statistics, vol.2, 3rd ed Griffin, London.

[14] Loschi, R.H. (1992). Coerência, Probabilidade e Calibração. , São Paulo, IMEUSP(tese de mestrado).

[15] Meinhold, R.J. and Singpurwalla, N.D. (1983). Understanding the Kalman Filter, American Statistican, Vol. 37, pp. 123-127.

[16] O'Hagan, Anthony (1994). Bayesian Inference, Cambridge.:Cambrige w University Press.

[17] Pereira, C.A.B. (1995). Estatística e Informação, Bolet.CL da ABE, Ano XI, 30, 1.

[18] Pereira, C.A.B. e Viana, M.A. (1982). Elementos de Inferência Bayesiana, V SINAPE.

[19] Wechsler, Sergio (1993). Exchangeability and Predictivism, Erkenntnis, 38, 343350 .

[20] Yule, G.U. and Kendall, M.G. (1945). An Introduction to the Theory of Statistics, 13 ed, London, Griffin. 